CERN-EP/2002-104

November 7, 2002

\title{
Performance of the ATLAS Electromagnetic Calorimeter End-cap Module 0
}

The ATLAS Electromagnetic Liquid Argon Calorimeter Group*

\begin{abstract}
The construction and beam test results of the ATLAS electromagnetic end-cap calorimeter preproduction module 0 are presented. The stochastic term of the energy resolution is between $10 \%$ $\mathrm{GeV}^{1 / 2}$ and $12.5 \% \mathrm{GeV}^{1 / 2}$ over the full pseudorapidity range. Position and angular resolutions are found to be in agreement with simulation. A global constant term of $0.6 \%$ is obtained in the pseudorapidity range $2.5<\eta<3.2$ (inner wheel).
\end{abstract}

(Submitted to Nucl. Instr. and Meth. A)

*) See next pages for the list of authors. 
B. Aubert, J. Ballansat, A. Bazan, B. Beaugiraud, J. Boniface, F. Chollet, J. Colas, P. Delebecque, L. Di Ciaccio, N. Dumont-Dayot, M. El Kacimi ${ }^{a}$, O. Gaumer, P. Ghez, C. Girard, M. Gouanère, H. Kambara, A. Jérémie, S. Jézéquel, R. Lafaye, T. Leflour, C. Le Maner, J. Lesueur, N. Massol, M. Moynot, L. Neukermans, P. Perrodo, G. Perrot, L. Poggioli, J. Prast, H. Przysiezniak, X. Riccadona, G. Sauvage, J. Thion, I. Wingerter-Seez, R. Zitoun,Y. Zolnierowski

Laboratoire de Physique de Particules (LAPP), IN2P3-CNRS, F-74019 Annecy-le-Vieux Cedex, France.

H. Chen, M. Citterio ${ }^{b}$, J. Farrell, H. Gordon, B. Hackenburg, A. Hoffman, J. Kierstead, F. Lanni, M. Leite ${ }^{c}$, D. Lissauer, H. Ma, D. Makowiecki, V. Radeka, D. Rahm, S. Rajagopalan S. Rescia, I. Stumer, H. Takai, K. Yip

Brookhaven National Laboratory (BNL), Upton, NY 11973-5000, USA.

D. Benchekroun, C. Driouichi, A. Hoummada, M. Hakimi

Faculté des Sciences Ä̈n Chock, Casablanca, Morocco.

R. Stroynowski, J. Ye

Southern Methodist University, Dallas, Texas 75275-0175, USA.

J. Beck Hansen, A. Belymam, J. Bremer, J.L. Chevalley, P. Fassnacht, F. Gianotti, L. Hervas, C.P. Marin, P. Pailler, P. Schilly, W. Seidl, J. Vossebeld, V. Vuillemin

European Laboratory for Particle Physics (CERN), CH-1211 Geneva 23, Switzerland.

A. Clark, I. Efthymiopoulos, L. Moneta

Université de Genève, CH-1211 Geneva 4, Switzerland.

B. Belhorma, J. Collot, A. Ferrari, M.L. Gallin-Martel, J.Y. Hostachy, P. Martin, F. Ohlsson-Malek, S. Saboumazrag

Institut des Sciences Nucléaires, Université Joseph Fourier, IN2P3-CNRS, F-38026 Grenoble, France.

J. Ban, N. Cartiglia ${ }^{d}$, H. Cunitz, J. Dodd, A. Gara, M. Leltchouk, S. Negroni, J.A. Parsons, M. Seman, S. Simion, W. Sippach, W. Willis

Nevis Laboratories, Columbia University, Irvington, NY 10533, USA.

F. Barreiro, G. Garcia ${ }^{e}$, L. Labarga, S. Rodier ${ }^{f}$, J. del Peso

Physics Department, Universidad Autónoma de Madrid, Spain.

C. Alexa ${ }^{g}$, P. Barrillon, C. Benchouk, A. Chekhtman, B. Dinkespiler, F. Djama, P.Y. Duval, F. Henrycouannier, L. Hinz ${ }^{h}$, M. Jevaud, P. Karst, A. Le Van Suu, L. Martin, O. Martin, A. Mirea ${ }^{i}$, E. Monnier, E. Nagy, D. Nicod, C. Olivier, P. Pralavorio, B. Repetti, M. Raymond, D. Sauvage ${ }^{j}$, S. Tisserant, J. Toth ${ }^{k}$, M. Wielers ${ }^{l}$

Centre de Physique des Particules de Marseille, Univ. Méditerranée, IN2P3-CNRS, F-13288 Marseille, France.

G. Battistoni, L. Carminati, G. Costa, M. Delmastro, M. Fanti, L. Mandelli, M. Mazzanti, G. F. Tartarelli

Dipartimento di Fisica dell'Universitá di Milano and INFN, I-20133 Milano, Italy. 
V. Aulchenko, V. Kazanin, G. Kolachev ${ }^{j}$, V. Malyshev, A. Maslennikov, G. Pospelov, R. Snopkov, A. Shousharo, A. Talyshev, Yu. Tikhonov

Budker Institute of Nuclear Physics, RU-630090 Novosibirsk, Russia.

E. Augé, C. Bourdarios, D. Breton, W. Bonivento ${ }^{m}$, P. Cros, C. de La Taille, I. Falleau, D. Fournier, G. Guilhem, S. Hassani, Y. Jacquier, K. Kordas ${ }^{i}$, G. Macé, B. Merkel, J.M. Noppe, G. Parrour, P. Pétroff, P. Puzo, J.P. Richer ${ }^{n}$, D. Rousseau, N. Seguin-Moreau, L. Serin, V. Tocut, J.J. Veillet, D. Zerwas

Laboratoire de l'Accélérateur Linéaire, Université de Paris-Sud, IN2P3-CNRS, F-91898 Orsay Cedex, France.

F. Astesan, W. Bertoli, A. Camard, B. Canton, S. Fichet, F. Hubaut, D. Imbault, D. Lacour, B. Laforge, O. Le Dortz, D. Martin, I. Nikolic-Audit, F. Orsini, F. Rossel, P. Schwemling

Laboratoire de Physique Nucléaire et de Hautes Energies, Université Paris VI et VII, IN2P3-CNRS, F-Paris, France.

W. Cleland, J. McDonald

Department of Physics and Astronomy, University of Pittsburgh, Pittsburgh, PA 15260, USA.

E.M. Abouelouafa, A. Ben Mansour, R. Cherkaoui, Y. El Mouahhidi, H. Ghazlane, A. Idrissi

Faculté des Sciences and Centre National de l'Énergie des Sciences et des Techniques Nucléaires, Rabat, Morocco.

J. Belorgey, R. Bernard, M. Chalifour, A. Le Coroller, J. Ernwein, B. Mansoulié, J.F. Renardy, J. Schwindling, J.-P. Taguet, J. Teiger

CEA, DAPNIA/Service de Physique des Particules, CE-Saclay, F-91191 Gif-sur-Yvette Cedex, France.

C. Clément, B. Lund-Jensen, J. Lundqvist, L. Megner, M. Pearce, S. Rydstrom

Royal Institute of Technology, Stockholm, Sweden.

J. Egdemir, R. Engelmann, J. Hoffman, R. McCarthy, M. Rijssenbeek, J. Steffens

State University of New York, Stony Brook, New York 11794, USA.

(a) Visitor from LPHEA, FSSM-Marrakech, Morroco.

(b) Now at Dipartimento di Fisica dell'Universitá di Milano and INFN, I-20133 Milano, Italy.

(c) Also at Sao Paulo University. Work partially supported by FAPESP/ Sao Paulo, Brazil.

(d) Now at Dipartimento di Fisica dell'Universitá di Torino, I-10125 Torino, Italy.

(e) Now at "Instituto Nicolas Cabrera", U.A.M. Madrid, Spain.

(f) Supported by the TMR-M Curie Programme, Brussels.

(g) Also at Institute of Atomics Physics, National Institute for Physics and Nuclear Engineering IFIN-HH, Bucharest, Romania.

(h) Now at Université de Lausanne, Faculté des Sciences, Institut de Physique des Hautes Energies.

(i) Now at University of Toronto, Dept. of Physics, Toronto, Canada.

(j) Deceased.

(k) Also at KFKI, Budapest, Hungary. Supported by the MAE, the HNCfTD (Contract F15-00), and the Hungarian OTKA (Contract T037350).

(1) Now at TRIUMF laboratory, Vancouver, Canada.

(m) Also at INFN, Sezione di Cagliari, Italy.

(n) Now at Institut des Sciences Nucléaires, Université Joseph Fourier, Grenoble, France.

This paper is dedicated to the memory of our colleague Dominique Sauvage, actively involved in the detector construction and beam test activities, who died accidentaly on March 16, 2002. 


\section{Introduction}

The electromagnetic calorimeter of the ATLAS experiment [1] consists of accordion-shaped lead absorbers interleaved with electrodes. The gap, filled with liquid argon (LAr), allows for the drifting of ionisation electrons under high voltage (HV). The calorimeter is composed of three parts, a cylinder (barrel) closed by two wheels (end-caps), and covers the pseudorapidity region $|\eta| \leq 3.2$. A general description of the complete ATLAS liquid argon calorimeter system can be found in [2]. This paper covers technical, construction and performance aspects of the end-cap full-size final module prototype (module 0 ) whose acceptance is $1 / 8^{\text {th }}$ of an end-cap wheel.

The main difficulty for the ElectroMagnetic End-cap Calorimeter (EMEC) is to accommodate the accordion geometry in the end-cap region $(1.375<|\eta|<3.2)$. The final design and the technical concepts chosen are based on the experimental results acquired during the R\&D programme and an intensive computer simulation effort [3]. Before starting full production, it was decided to build and expose to particle beams a module 0 . The main difference with the end-cap prototype described in [4], apart from the size and the presence of a section with $\eta$-strips (to improve the position and angular resolution), is the choice of a constant lead thickness for the absorbers, more convenient for economical and technical reasons [5]. As in ATLAS, a presampler (PS) device is placed in the region $1.5<\eta<1.8$, in front of the calorimeter.

The paper is organised as follows. Section 2 and 3 are devoted to module and presampler construction. Section 4 presents the experimental beam test setup. The signal reconstruction and performance of the ATLAS-like electronics chain are described in sections 5 and 6 , followed by the presentation of the beam test results in section 7 . Section 8 contains the conclusions for the module 0 and prospects.

\section{Calorimeter Construction}

The electromagnetic end-cap calorimeters consist of 2 wheels, one on each side of the electromagnetic barrel. Each wheel, divided into eight wedge-shaped modules, is $63 \mathrm{~cm}$ thick with internal and external radii of about $30 \mathrm{~cm}$ and $2 \mathrm{~m}$, respectively. The absorber plates are mounted in a radial arrangement like the spokes of a bicycle wheel, while the accordion waves are parallel to the front and back edges of the wheel and run in depth, as can be seen on Figure 1. To ensure a good azimuthal uniformity of the response, the combined thickness of liquid argon and absorber crossed by the particles must be independent of the azimuth angle $(\phi)$. Therefore the liquid argon gap increases with the radius, and it is necessary to vary the wave amplitude and the folding angle at each radius. For feasibility reasons, the folding angle must be kept between $60^{\circ}$ and $120^{\circ}$. As it was impossible to fulfil this requirement in covering the full pseudorapidity range with only one accordion structure, each end-cap calorimeter consists of two coaxial wheels. The boundary between these two wheels is projective $(\eta=2.5)$. The corresponding crack is about $3 \mathrm{~mm}$ wide and mainly filled with low density material. In total, there are 768 absorbers in the outer wheel and 256 in the inner wheel.

The detector signal is proportional to the sampling fraction and the drift velocity, and inversely proportional to the liquid argon gap thickness. As the variation of the gap and of the sampling fraction along the radius partially compensate each other, an $\eta$-independent detector response can be achieved with an electric field strength almost independent of the radius. Figure 2 shows the associated variation of the high voltage with respect to $\eta$ (open circles). However, for technical reasons, a high voltage varying by steps (solid triangles on Figure 2) was chosen. The outer (inner) wheel, is divided into seven (two) high voltage sectors.

Over the region devoted to precision physics $(1.5<\eta<2.5)$, the detector is segmented into three longitudinal sections. The first section, narrow strips, ends after about 4 radiation lengths $\left(X_{0}\right)$, while the second section stops after a total of about $22 X_{0}$. The back section adds from 4 to $12 X_{0}$ depending on $\eta$. The inner wheel electrode contains only two longitudinal sections, separated after $22 X_{0}$, resulting in a total number of $X_{0}$ between 26 and 34. The segmentation of the EMEC is summarised in Table 1. There are 3888 readout cells per module.

\subsection{Absorbers}

The absorbers are made of lead plates $(1.7 \mathrm{~mm}$ and $2.2 \mathrm{~mm}$ thick for the outer and inner wheel, respectively) cladded with two layers of $0.20 \mathrm{~mm}$ thick stainless steel to ensure the absorber rigidity and smooth surface for high voltage. These layers are glued using a $0.15 \mathrm{~mm}$ thick glass-fibre prepreg adhesive. The absorbers have nine waves in the outer wheel and six waves in the inner wheel. Along the two straight edges of the folded absorbers, corresponding to the front and back sides of the end-cap calorimeter, two longitudinal glass-epoxy bars are glued. To increase the absorber rigidity, a bar transverse to the waves is glued on the edge at large radii of each absorber. These bars are used to position the absorbers with 
a precision of the order of $0.1 \mathrm{~mm}$ during stacking. The weight of an outer (inner) wheel absorber is approximately $26 \mathrm{~kg}(8 \mathrm{~kg})$.

No major problems were encountered during the absorber fabrication ${ }^{1)}$ : out of the 100 (36) outer (inner) absorbers produced only 2 could not be used since they were damaged during their manufacture. Each absorber is controlled by optical inspection, as well as by thickness and width measurements at predefined positions. For $10 \%$ of the absorbers, a 3D measurement is also performed to detect eventual changes of the geometry during mass production.

To minimise the contribution of passive material to the constant term in the energy resolution, stringent tolerances on the lead plate thickness and the liquid argon gap must be imposed. Simulation indicates that a thickness distribution with a r.m.s. less than $17(22) \mu \mathrm{m}$ in the outer (inner) wheel is required to achieve a contribution not larger than $0.3 \%$ to the local constant term [2]. All the plates were thickness mapped, using a technique based on ultrasounds [6]. A r.m.s. of $9 \mu \mathrm{m}$ for all the measurements is obtained, well below the tolerance. The liquid argon gap must be uniform at the $2 \%$ r.m.s. level which implies geometrical tolerances on the produced bent absorbers. As an example, Figure 3 shows the distribution of the measured thicknesses at both edges (small and large radius). Their r.m.s., 28 and 35 $\mu \mathrm{m}$ respectively, have to be compared with the estimated tolerances of 110 and $36 \mu \mathrm{m}$. The non Gaussian shape of the distributions comes from the fact that the absorbers tend to be systematically thinner at their front and back sides.

\section{$2.2 \quad$ Electrodes}

The readout electrodes [7] are flexible printed circuit boards folded with the same geometry as the absorbers. They consist of three layers of copper, insulated by two Kapton polyimide sheets: the external layers provide the high voltage to the liquid argon gaps, while the internal layer allows the signal collection by capacitive coupling. For each layer, projective towers are defined in $\eta$ by copper etching. These electrodes are similar to the ones in the barrel electromagnetic calorimeter. Material characteristics, electrode production and test procedures may be found in [8].

Figure 4 shows an electrode before bending used to equip the outer wheel. The resistive ink pads of the high voltage layer, visible on this picture, have to minimise the crosstalk between neighbouring channels and protect the front-end electronics against sparking, which would require high resistance values. They also have to minimise the high voltage drop induced by the minimum bias pile-up events which would require low resistance. A compromise is reached with a nominal resistance value of $1 \mathrm{M} \Omega / \mathrm{square}$ (at room temperature) which maintains a relative drift velocity variation at the level of $0.1 \%$, and consequently a negligible contribution to the constant term [9],[10]. In the outer wheel, signals from the first longitudinal section are routed to the front side of the detector. Except for $\eta<1.5$, signals from both the second and third sections are routed to the back face (as well as in the inner wheel). The connection striplines of the second section are designed to minimise the electrode inductance (kept constant at about $17 \mathrm{nH}$ ). Output connectors are stapled and soldered on both flat edges of the electrodes. A coppered kapton foil (flap) glued on a cylindrical plastic tube, is soldered on the ground pad of the electrode to ensure grounding of the absorber.

The bending machine is a single knife associated with a press, adapted to obtain a variable folding angle [11]. The knife pushes the electrode against an elastic support (foam plate), so that the electrode is bent without longitudinal stress, and the fold is symmetrical with respect to the knife axis. To ensure an optimal geometry, this method requires a precise positioning of each fold before bending. This is achieved with two rectangular indexing holes per fold, machined in extra material added on both edges of each electrode (see Figure 4). This extra material is removed after bending.

Electrodes were produced by two suppliers ${ }^{2)}$ : after high voltage tests and resistor measurements, 48 bent electrodes were accepted for the outer wheel and 31 for the inner wheel.

\subsection{Honeycomb Spacers}

The spacers keep the electrode centred in between the absorbers. They consist of honeycomb strips, made of NOMEX impregnated with phenolic resin, laid in the flat parts of the accordion. The strips are cut from plates produced with linearly variable thickness, with a tight tolerance of $+0.05 \mathrm{~mm}$ and -0.25 $\mathrm{mm}$.

Two honeycomb strips are used to cover the overall length of the absorber. Even if the width of the flat parts of the accordion varies as a function of the radius, it is kept constant to ease the honeycomb strip cutting. The strips are linked together with flexible glass-fibre wires glued only in contact to the

\footnotetext{
1) FIBERTECNIC SA Industrie, P. de Gamarra 40, Apdo. 85, 01013 Vitoria (Spain).

2) Cicorel SA, 8 route de l'Europe, CH-2017 Boudry; MCB Industrie, 107-11 rue du Moulin Sarrazin, F-95100 Argenteuil.
} 
honeycomb material. The relative position of the strips is given by jigs used to have the glue set. Flexible nets are obtained which are easy to handle and put in place during the stacking of absorbers and electrodes.

\subsection{Module Assembly}

Given the limited number of accepted outer wheel electrodes at that time, it was not possible to build a fully equipped module. To reduce edge effects, these electrodes were placed in the central region of the module. In total, the outer wheel contained half of the electrodes (48) but all the absorbers (96), while the inner wheel was complete (32 absorbers and 31 electrodes).

The stacking is performed in a clean room, sealed from external air penetration, with a small atmospheric overpressure and a constant temperature of $22^{\circ} \mathrm{C}$. Humidity is not regulated but only controlled. Figure 5 shows the support structure where the module is stacked. The total weight of the detector is supported mainly by two large external aluminium rings. However, in order to minimise the width of the crack between the outer and inner wheels and link these wheels together, intermediate rings are used at $\eta=2.5$ (the front one is made of composite material to reduce the amount of dead material in front of the detector). During stacking, five lighter aluminium rings are added: two on each side of the outer wheel to control the gap thickness, and one on the circumference in between the two external rings to minimise the absorber deformation.

The stacking operation starts by handling one large absorber, previously clea-ned, with a lifting beam equipped with suckers. The heads of the longitudinal bars are inserted into the grooves of the rings, and slide down along them. The position of each absorber is then precisely defined by inserting pins and screws into centering holes drilled in both absorber bars and support rings. A first layer of honeycomb spacers is then put in place. After cleaning with alcohol, the electrode is installed and kept in position by using the readout summing boards (see section 2.5). These boards compensate the spring strength of the flaps and ensure that the first and last folds of the electrode are parallel to the longitudinal bars. A second layer of spacers is put on the electrode and the complete gap is closed with the next absorber.

To control the bulging of every absorber, the relative height of each wave along four (one) equidistant lines, orthogonal to the front and back sides of the module, in the outer (inner) wheel is measured. A high voltage test of the electrode is performed using a power supply with a current sensitivity of 1.0 $\mu \mathrm{A}$ (the high voltage values given in Figure 2 are reduced by a factor 1.5). When this electrical test is not successful, all components are unstacked, cleaned (or replaced) and stacked again. On average, four complete detection gaps were stacked per day.

Figure 6 shows the sagging evolution during stacking, on the central wave, for a line around $\eta=1.8$. Two different slopes are observed: $i$ ) $0.4 \mathrm{~mm}$ per absorber before the $13^{\text {th }}$ absorber and $\left.i i\right) 0.05 \mathrm{~mm}$ for the rest of the stacking operation. They correspond to 2 phases of stacking: $i$ ) no mechanical constraints are applied on top of the absorber and $i i$ ) the absorbers are mechanically stressed by putting some loads $(50-100 \mathrm{~kg})$.

A global high voltage test was performed on all electrodes after stacking completion. Several problems, not observed during stacking, were discovered in the outer wheel during this test. They are presented in Figure 7. To meet the beam test schedule, it was not possible to dismount the detector to try to solve these problems, and so all the faulty high voltage channels were disconnected.

\subsection{Module Cabling}

Two types of boards made of multi-layer PCBs are plugged on the electrode connectors. The first one, called HV board, distributes the high voltage to $\phi$-sectors of 24 and 8 electrodes in the outer and the inner wheel, respectively. There are two HV cables, soldered on the board, to supply the lower and upper gaps separately. The second type, the summing board (SB), performs the signal summation in $\phi$ defining the granularity (Table 1): 12 adjacent electrodes are summed for a front cell and 3 (4) for middle and back cells in the outer (inner) wheel.

Motherboards (MB) are plugged on top of the summing boards. They ensure the signal routing and the distribution of calibration signals through resistive networks. A high inductive crosstalk, observed on the back side boards, will be discussed in detail in section 6.2.

Figure 5 shows the back side of the electromagnetic end-cap module 0 with its cold electronics (SB, HV boards and MB), inside the stacking frame in the clean room. The readout cable harnesses, 64 channel kapton coaxial cables, are plugged on the motherboard and routed out of the module. To reduce the noise contribution, cables with $25 \Omega$ and $50 \Omega$ characteristic impedance are used for the middle/back and front sections, respectively. 


\subsection{Capacitance and Inductance Measurements}

Measurements of detector cell capacitances $\left(\mathrm{C}_{t}\right)$ and inductances $(\mathrm{L})$ were carried out using an RLC-meter at the summing board level. Figure 8 shows a comparison between measured and theoretical $\mathrm{C}_{t}$ and $\mathrm{L}$ in the middle section, as a function of $\eta$. Theoretical expectations come from the electrode modeling where the capacitance is computed as the sum of the capacitance to the grounded absorber and the coupling capacitance to neighbouring cells in $\eta$. Contribution from the summing board is not added which may explain the slight difference with the data. The variation of the capacitance reflects the gap and cell size variation with $\eta$. The measured inductance exhibits a pattern with a periodicity of 4 cells (corresponding to a change of summing board), not expected by the electrode modeling. This is traced back to an insufficient grounding both on the electrode and on the summing board and corrected on production modules. A variation of inductance versus $\phi$ is also observed. This effect, less than $8 \mathrm{nH}$, is due to differences in summing board stripline lengths.

\section{Presampler Construction}

The end-cap presampler is placed in front of the electromagnetic end-cap calorimeter in the region $1.5<\eta<1.8$, resulting in a radius ranging from $1702 \mathrm{~mm}$ to $1232 \mathrm{~mm}$. It is divided into 768 cells (per end-cap wheel) of $\Delta \eta \times \Delta \phi=0.025 \times 2 \pi / 64$, and 32 identical azimuthal sectors (modules). The isometric view of the end-cap presampler module is shown in Figure 9.

Each module consists of two $2 \mathrm{~mm}$ thick active liquid argon layers, formed by three electrodes parallel to the front face of the EM end-cap calorimeter. The electrodes, made from double sided printed circuit boards, are separated by honeycomb spacers and glued together at the inner and outer radius with $2 \mathrm{~mm}$ thick G10 bars. A negative high voltage is applied to the external electrodes and the signals are read out from the central electrode segmented into pads. The capacitances of readout cells decrease with $\eta$ from $122 \mathrm{pF}(\eta=1.5)$ to $69 \mathrm{pF}(\eta=1.8)$. The signal pads are connected by pins to the $50 \Omega$ striplines, printed on the middle layer of the top external electrode, and finally readout at the outer radius of the module by $50 \Omega$ impedance cables. The presampler design is sketched in Figure 9 .

The integrity of the electrode signal and HV lines are electrically tested before and after the assembly, at room temperature. Their capacitances are also measured. After the module assembly, similar tests are repeated in liquid nitrogen and after warm-up. No problems were encountered for the two fabricated presampler modules.

\section{$4 \quad$ Experimental Beam Test Setup}

The calorimeter and presampler modules were inserted in the cryostat of the former NA31 experiment [12], and tested during three weeks at the CERN H6 beam line (North Hall Experimental Area). The cryostat is connected to a common cryogenic system used by the ATLAS liquid argon community [13]. Before its insertion in the cryostat, the detector is attached to its top flange in a clean room. The presampler modules are mechanically fixed to the front side of the calorimeter. The EMEC and PS readout and HV cables are connected to the cryostat harnesses. These signals are extracted through a high density pin carrier from the cryostat to the cover plate of the cryostat and connected to the front end crate by strip-lines. The module is then inserted in the cryostat which is closed, cooled down with liquid nitrogen heat exchanger, in a gas phase above the calorimeter ( $2 \mathrm{~K}$ per hour), and filled with liquid argon. The argon pressure is controlled with a precision of a few per mil leading to negligible temperature variations with time. The presence in the argon of electro-negative impurities $\left(\mathrm{O}_{2}, \mathrm{CO}_{2}, \ldots\right)$, which may trap electrons drifting in the electric field, is measured to be less than $0.1 \mathrm{ppm} 0_{2}$ equivalent.

\subsection{Beam Line Instrumentation}

Figure 10 shows the $\mathrm{H} 6$ beam line instrumentation. The trigger is defined by the coincidence of a wide area scintillator (S1), 18 meters upstream from the cryostat, plus two narrow ones (S2 and S3), located close to the entrance face of the cryostat. In addition, the analog signal recorded on $\mathrm{S}_{\pi}$ (placed just behind the cryostat) is used offline to flag pions, while muons can be vetoed in the trigger by $\mathrm{S}_{\mu}$ (located after the dump), to increase the electron purity. The particle path is reconstructed using 4 beam chambers [14], readout by delay lines. To improve the position reconstruction, constant fraction discriminators are used.

Electron/positron secondary and tertiary beams are derived from SPS protons and their energies $\left(\mathrm{E}_{\text {beam }}\right)$ range from 10 to $200 \mathrm{GeV}$. The beam is tuned to obtain a good particle rate (typically 500 events are recorded per burst when muons are vetoed) and low pion contamination. The beam spot size lies between 1 and $3 \mathrm{~cm}^{2}$ depending on the energy, which is insufficient to completely cover a cell size (especially at low $\eta$ ). 
As the beam direction is fixed, the module is scanned by moving the cryostat in $\eta$, and the detector in the liquid argon in $\phi$. In both case, the projectivity of the detector is kept. The dead material thickness in front of the active part of the calorimeter (cryostat walls, cold electronics, cables, liquid argon, ...) is almost constant with $\eta$ and amounts to $1.7 \pm 0.1 X_{0}$. In the region $1.5<\eta<1.8$, the presence of the presampler device adds a thickness of the order of $0.1 X_{0}$.

\subsection{Electronics and Data Acquisition}

On top of the cryostat, the front-end ATLAS-like electronics is located in two crates which can house up to 38 Front End Boards (FEBs) and two calibration boards, which deliver $0.2 \%$ amplitude accuracy input pulses. A detailed description of the electronics readout can be found in [15]. The FEBs (128 channels each) amplify, shape and store the analog signals coming from the mother boards in the Switch Capacitor Array at a $40 \mathrm{MHz}$ frequency during the trigger latency. To cope with the large dynamic range, a three gain shaper is used (ratio 1:9.2:92 called high, medium and low gain), but with the beam energy available, only high and medium gains are relevant. For each trigger, 5 samples for each of the 2 gains are digitised for a given channel directly on the FEB and these signals are sent to the MiniRODs (16 parallel FIFOs per FEB) in the electronics barrack through 64 channel copper cables. At the end of a beam burst, all the events stored in the MiniRODs are emptied by a RIO ${ }^{3)} 8062$ processor.

The Data Acquisition system (DAQ), based on the RD13 data flow protocol [16], performs the event building using informations coming from the beam chambers, some scintillators and the calorimeter. Since the beam is asynchronous (unlike in ATLAS), the phase $(t d c)$ between the trigger and the next leading edge of a $40 \mathrm{MHz}$ clock serves as time reference for the digitised samples.

Pedestal runs of 2000 events are regularly taken. Two kinds of calibration runs are recorded: delay runs, for which the calibration board generates pulses with a fixed amplitude input value and a variable delay time for the pulse start, and ramp runs, for which the calibration board generates pulses with linearly increasing input amplitude and a fixed time for the pulse start. The first type of run allows to reconstruct the signal pulse shape of each cell, whereas its gain coefficient can be computed with the second type. For runs with electron beam, 20000 events are recorded using two gains (high and medium) per event, including typically 500 random triggers. For each of these runs, the data quality is monitored, and databases are created online for the pedestal values.

\section{$5 \quad$ Signal Reconstruction}

The amplitude of the response shape is computed using an optimal filtering technique [17]. In this method, maximum amplitude $\left(A_{\max }\right)$ and difference between the assumed and reconstructed time $(\Delta t)$ are linear combinations of samples $\left(S_{i}\right.$, in ADC units, after pedestal subtraction):

$$
A_{\max }=\sum_{i=1}^{n} a_{i} S_{i}, \quad A_{\max } \Delta t=\sum_{i=1}^{n} b_{i} S_{i}
$$

Parameters $a_{i}$ and $b_{i}$ are sets of coefficients computed for each phase by bins of 1 ns. They are determined using an analytical parametrisation of the response pulse and its derivative, for both calibration and physics signals. The computation of $a_{i}$ and $b_{i}$ parameters takes into account the autocorrelation between all samples, computed in pedestal runs. The number of samples, $n$, is fixed to 5 . The value of $A_{\max }$ is determined after an iterative process until the convergence criteria is satisfied $(|\Delta t|<1 \mathrm{~ns})$, which is the case after less than 4 iterations.

To parametrise calibration and physics pulse shapes a first try was made using an electrical model of the detector-preamplifier-shaper system. The shape of the response pulse to a calibration signal is reconstructed for each channel, with a 1 ns step size using delay runs and subtracting the calibration parasitic injected charge. The physics pulse shape could be predicted using the capacitance and inductance deduced from calibration. The drift time is left as a free parameter. However, this approach suffers from a high sensitivity to reflections in the signal path, and can not properly reproduce the rising edge of the pulse (most sensitive to the inductance value) [18].

To overcome the above difficulties, a function that fits shapes, regardless of their physical meaning, is used to compute the optimal filtering coefficients for the data analysis. The shapes of each calibration pulse and its derivative are tabulated. For physics events, since data were taken in asynchronous mode, pulse shapes are reconstructed with a time binning of $1 \mathrm{~ns}$ using the time $t d c$. To minimise the distortion induced by the crosstalk, runs with the highest energy beam are used, and only cells with sufficiently high energy deposit are considered. To obtain a complete map of the detector, $\phi$ symmetry is assumed

3) CES, 38 avenue Eugène-Lance, PO Box 584, CH-1212 Grand-Lancy 1. 
for the non covered cells. Physics shapes are then fitted using neural network techniques [19]. Figure 11 shows the result of the fit for one channel in the middle section. The residuals are kept below \pm $2.5 \%$. Calibration and physics events differ by their input signal shapes (exponentially decreasing and triangular, respectively) and the injection point of these signals. As it is shown in Figure 12, the latter point generates a different bias on the physics and calibration pulse [15]. However the needed correction factor is not applied here, mainly because of the high level of inductive crosstalk which can not be removed in the procedure.

\section{$6 \quad$ Performance of the Electronics Chain}

To correct for the gain dispersion of the electronics chain and study the stability over time for all the detector cells, ramp runs were regularly taken (typically every 24 hours). In these runs, channels are pulsed by calibration patterns (4 per section) with 11 input calibration voltages (DAC units) of increasing amplitudes. The cell response dependence to the input amplitude is fitted by a second order polynomial, in high and medium gains. For instance at $\eta=1.6$, the integral non linearity in the medium gain for the middle section is about $0.3 \%$, while the linear term is around $15 \mathrm{MeV} / \mathrm{ADC}$ counts.

The conversion factor from $\mathrm{DAC}$ units to energy in $\mathrm{GeV}$ is computed using the beam test data and compared to the one predicted by the simulation, which include the full geometry description and electric field maps. The difference is of the order of $10 \%$, giving the accuracy of the prediction of the absolute energy scale. This value is higher than in the barrel calorimeter [15] because of the variation of the cluster containment and high voltage as a function of the rapidity.

To estimate the stability of the detector response with time, the energy variation (for a given value in ADC counts) for each channel of a given FEB is computed. Figure 13 shows the normalised result $(\Delta E / E)$ as a function of time for the different compartments. The maximum variation over the whole run period is around $0.3 \%$.

\subsection{Noise}

The noise study is carried out using data from pedestal runs. Using optimal filtering coefficients, the noise reduction factor (specific to beam tests during which there is no pileup) varies from 1.7 to 1.35 compared to a single sample measurement, depending mainly on the cell capacitance. Figure 14 shows the noise per cell (average in $\phi$ ) as a function of $\eta$ for the PS and each layer of the EMEC. The coherent and the incoherent noise are deduced from the sum and alternate sum of the 128 channel pedestal values (corresponding to one FEB), respectively. The coherent/incoherent noise ratio is found to be around $10 \%$.

To reduce the noise contribution, a software gain selection is used, i.e. the high gain is chosen only if a predefined sample is below a given threshold, defined to avoid saturation effects.

\subsection{Crosstalk}

A complete and detailed map of the crosstalk in the EMEC is obtained using the delay runs [20]. Two quantities are used: the amplitude of the crosstalk at its peak and at the signal peak (relevant for physics). Both quantities are divided by the maximum signal amplitude of the pulsed channel.

The main contribution, expected from analytical computation [21], comes from the capacitive coupling between detector cells. Figure 15 shows the crosstalk values in the front section and a crosstalk shape which is almost the first derivative of the pulse, due to the capacitive coupling. The decrease from $8 \%$ to $5 \%$ in the strip region $(1.5<\eta<2.4)$ for the peak to peak crosstalk as well as the crosstalk shape are well reproduced by the simulation, and are explained by the decrease of the coupling capacitance value (50 to $30 \mathrm{pF}$ for a typical cell capacitance above $100 \mathrm{pF}$ ). An additional capacitive component coming from the summing board explains the dip seen each 8 or 12 cells depending on the granularity. A resistive crosstalk, below $0.15 \%$, between front and middle sections is also observed, due to the HV ink resistors on the electrode.

Figure 16 shows the crosstalk in the middle section. In this case, the crosstalk shape is almost a second derivative of the signal pulse, showing the large contribution from the inductive coupling. Testbench measurements demonstrated that summing and mother boards are responsible for most of this crosstalk. Some correlations exist between the crosstalk amplitude and the inductance measured on the module (Figure 8b). Similar values are observed for the back cells and between middle and back cells. The crosstalk between front and back sections, which comes from the electronic chain after the mother board, is of the order of $0.1 \%$.

To reduce the high inductive crosstalk on the cold electronics, mother and summing boards have been redesigned. A reduction by a factor of 2 is achieved, leading to an acceptable contribution (Figure 16 ), and the crosstalk between middle and back section is completely removed. 
Response to Electrons

A total of 18 million of events were recorded. For electron energy beams lower than $80 \mathrm{GeV}$ the electron sample represents $75 \%$ of the recorded events. For higher energies, the beams are more polluted with pions, and the electron purity drops to $50 \%$.

\subsection{Electron Reconstruction}

The electron energy is reconstructed by summing the clusters formed in each compartment around the cell with maximum energy deposit. In the presampler, the best compromise between signal and noise, deduced from the beam test data, is found to be 3 cells in $\eta$. In the front section, an $\eta$ cluster size around \pm 0.03 is chosen, as suggested by simulation. Because of changes in the granularity, this corresponds to 23 , 15,11 and 3 strips in the regions $[1.5,1.8],[1.8,2.0]$ and $[2.0,2.4],[2.4,2.5]$ respectively. The cluster size in $\phi$, for the presampler and the front section, is 1 or 2 cells depending if the shower develops near or far from the center of the cell. In the middle section of the outer wheel, the cell size decreases continuously with $\eta$. A cluster with 5 cells in $\eta$ and $\phi(5 \times 5)$, proven to minimise the energy leakage at high $\eta$, is used for uniformity studies in $\eta$, while a $3 \times 3$ cluster is chosen for the energy scans in the region $[1.5,1.8]$. In the inner wheel, a $3 \times 3$ cluster size is chosen. In the back section, because of the high level of crosstalk between middle and back cells (section 6.2), only one cell is forming the cluster, the one behind the middle cell with maximum energy deposit. The cluster energy in the back section is used to compute the total energy only if $\mathrm{E}_{\text {beam }}>50 \mathrm{GeV}$. Table 2 summarises the different cluster sizes used in this analysis. To estimate the impact position of the particle in the calorimeter, energy weighted barycenters are computed in the front section for $\eta$ (except in the inner wheel) and in the middle section for $\phi$. The presampler, located in the region $[1.5,1.8]$, is only used for a dedicated study with additional dead material in front of the calorimeter (section 7.2).

Because of the end-cap geometry and the choice of projective HV sectors (section 2), the total energy $\left(E_{t o t}\right)$ increases with $\eta$ inside each HV sector $(l)$ according to the simplified expression:

$$
E_{\text {tot }} \sim \frac{f_{\text {samp }}}{g} v_{d} \sim \frac{f_{\text {samp }}}{g} E_{\text {field }}^{b} \sim \frac{f_{\text {samp }}}{g^{1+b}} U^{b}
$$

where $g$ is the liquid argon gap thickness, $f_{\text {samp }}$ the sampling fraction, $v_{d}$ the drift velocity, $E_{\text {field }}$ the electric field in the gap, and $U$ the HV value. Figure 17 shows the energy dependence on high voltage in the region $1.6<\eta<1.8$ and the fitted value of the $b$ parameter. To correct for the slope of the energy response inside HV sectors, $\eta$-dependent weights $\left(w_{l}\right)$ are applied on each cell [22]:

$$
w_{l}\left(\eta^{j}\right)=\beta_{l}\left(1+\alpha_{l} \cdot\left(\eta^{j}-\eta_{l}\right)\right)
$$

where $\eta_{l}$ and $\eta^{j}$ are the $\eta$ values at the center of the high voltage sector $l$ and at the center of the middle cell $j$, respectively. $\alpha_{l}$ parameters are obtained with the beam test data by minimising the total energy dispersion inside each high voltage sector. As an example, Figure 18 shows the result for the region $1.6<\eta<1.8$. To take into account effects due to inaccurate high voltage setting in each sector, a global factor $\left(\beta_{l}\right)$ is also applied. A comparison with the simulation expectations is shown in Figure 19. Discrepancies between beam test data and simulation for $\alpha_{l}$ parameters are attributed to the absence of a second ground return and the high value of the inductive crosstalk.

Because of the finite size of the cluster, the energy depends on the beam impact point inside the cell, which can be estimated by the $\eta$ and $\phi$ barycenters. In the $\eta$ direction, this dependence is fitted by a parabola and corrected. In $\phi$, the effect is convoluted with the local non-uniformities of electric field and sampling fraction, due to the accordion geometry. Figure 20 shows the shapes of $\phi$-modulations in absorber unit at different $\eta$ for a $100 \mathrm{GeV}$ beam. Simulation results are superimposed and a good agreement is found. The variation of the modulation amplitudes, from $1 \%$ to $4 \%$, is the consequence of the folding angle and the gap dependence with $\eta$. They are parametrised with Fourier series [23]:

$$
F(\Phi)=a_{0} \cdot\left(1+\sum_{k=1}^{3} a_{k} \cdot \cos (2 \pi k \Phi)+b_{1} \cdot \sin (2 \pi \Phi)\right)
$$

where $\Phi$ is in absorber unit $(-0.5<\Phi<0.5), a_{0}$ is the mean total energy and $a_{k}$ are the even coefficients. $b_{1}$ is an odd coefficient which takes into account calorimeter (or calibration) local defects. Fitted parameters of $\phi$-modulations versus $\eta$ are shown in Figure 21. Cosine parameters $\left(a_{k}\right)$ vary smoothly and can be fitted with a parabola for $a_{1}$ and $a_{2}$ and a straight line for $a_{3}$. The sine parameter $\left(\mathrm{b}_{1}\right)$ is small in 
amplitude compared to $a_{1}$ and $a_{2}$ and compatible with 0 . Similar results are obtained in the inner wheel but the variation of $\phi$-modulation parameters is much smaller.

As an example, Figure 22 shows the total energy distribution at $\eta=1.9$ after $\mathrm{HV}$ and geometrical corrections. These corrections improve the energy resolution $\left(\sigma_{E}\right)$ by $12 \%$. For all results presented in the following, the energy resolution and the total energy are extracted from a Gaussian fit starting $1.5 \sigma$ below the mean energy (the low tail side of the distribution is non Gaussian because of the electron bremsstrahlung contribution).

\subsection{Energy Resolution}

Nine positions were chosen in the calorimeter to perform the energy scans. After all corrections are applied, the energy resolution is computed and noise and beam energy spread (generally negligible) are subtracted. The noise contribution is estimated using random triggers at each point and for each energy value. As an example, a noise of $190(210) \mathrm{MeV}$ at $10(180) \mathrm{GeV}$ is obtained in a $5 \times 5$ cluster at $\eta=1.9$. The noise slightly increases with $\eta$ in the outer wheel as the number of cells in medium gain increases (because of the smaller cell size). The energy resolution is parametrised at each point as:

$$
\frac{\sigma_{E}}{E}=\frac{a_{E}}{\sqrt{E}} \oplus b_{E}, \quad E(\mathrm{GeV})
$$

where $a_{E}$ is the sampling term, $b_{E}$ the local constant term. As an example, Figure 23 shows the result obtained at $\eta=1.9$ for the beam test data and the simulation. The small differences observed are attributed to the smaller beam spot size at high energy $(\mathrm{E} \geq 150 \mathrm{GeV})$, resulting in an underestimated constant term for the beam test data (the beam spot size is the same at all energies in the simulation).

Figure 24 summarises the results. The stochastic term reaches its minimum around $\eta=1.9$ in the outer wheel, as expected by the simulation. This corresponds to the region where the sampling fraction is still high (around 22\%) and the $\phi$-modulation amplitudes already low (less than 1\%). The constant term is below $0.4 \%$, compatible with expectations, except for the positions located near a transition region between two $\mathrm{HV}$ zones where it rises up to 0.5-0.6\%.

For dedicated runs, steel plates (with depth $\mathrm{d}_{m}$ in $\mathrm{X}_{0}$ units) have been put in front of the cryostat to obtain an ATLAS-like configuration $\left(2\right.$ to $3 X_{0}$ for $1.55<\eta<1.8$ ). Figure 25a shows the presampler energy weight $\left(\alpha_{\text {pres }}\right)$ optimisation for a $60 \mathrm{GeV}$ energy beam and $1.8 \mathrm{X}_{0}$ additional dead material in front of the cryostat. Using this optimised energy weight for the presampler, the energy resolution is largely improved, as shown in Figure 25b. More generally, an empirical formula $\alpha_{\text {pres }}=4.9 \times\left(\mathrm{d}_{m}+1.7\right)$ is used for this weight in the following, whatever the beam energy. It reflects the 'sampling fraction' of the presampler for the dead material in front of it. Overall results for energy scans with different amounts of additional dead material are presented in Figure 26. The stochastic term of the energy resolution is worse by a factor of 1.3 and 2 when $1.8 X_{0}$ and $2.7 X_{0}$ is added in front of the cryostat, respectively. The non-linearity of the detector response at $\eta=1.7$ is around $1 \%$ for $\mathrm{E}_{\text {beam }} \geq 40 \mathrm{GeV}$. When $2.7 X_{0}$ of additional material is added, it rises to $2 \%$. A fair agreement is found for these results with the Monte Carlo when $\mathrm{E}_{\text {beam }}>40 \mathrm{GeV}$. The simultaneous optimisation of presampler and calorimeter longitudinal section weights does not improve the energy resolution significantly [24].

\subsection{Study of the Crack Region}

The crack region $(2.45<\eta<2.55)$ was scanned at fixed $\phi$. The interleave $(3 \mathrm{~mm})$ between the two wheels is filled with the G10 transverse bars of the inner wheel absorbers, which represents around $3 X_{0}$ in depth, to be compared to $38 X_{0}$ in the outer wheel (at $\eta=2.49$ ) and $28 X_{0}$ in the inner wheel (at $\eta=2.51$ ). It should be noted that inner and outer wheel electrodes are not coppered on the last $1.5 \mathrm{~mm}$ near $\eta=2.5$ for technical reasons, which affects the charge collection in this region.

For this study, a constant cluster size including cells of both wheels is chosen for the energy reconstruction [25]. Figure 27 shows the total energy and the energy resolution as a function of $\eta$. Between the inner and outer wheel, the electron energy is affected only in a small zone of about $10 \mathrm{~mm}$, where up to $60 \%$ of the total energy is lost. The presence of remaining pions (not completely removed by the scintillator cut) and the behaviour of the innermost middle cell of the outer wheel (large inductance, low capacitance) may explain the degradation of energy resolution in the outer wheel. In the inner wheel, the energy resolution is as expected from simulation.

\subsection{Uniformity of the Inner Wheel}

Because of the problems encountered during the stacking (section 2.4), no relevant results concerning the uniformity could be obtained in the outer wheel. But in the inner wheel, all cells were scanned with a $100 \mathrm{GeV}$ energy beam, except for cells where part of the electromagnetic shower starts to leak outside the 
detector. Average $\phi$-modulation corrections (for a given $\eta$ value) are computed and applied to all cells. Because of the cell size decrease with $\eta$, sizeable leakage outside the cluster in $\phi$ is observed for $\eta>2.9$, and corrected with a parabola shape as a function of $\phi$.

The overall energy spectrum, with half a million entries, is shown in Figure 28 after all corrections are applied. Subtracting the contributions from the average sampling term $(1.05 \%)$, the noise term $(0.5 \%$, because only medium gain is used in this study) and the beam energy spread $(0.2 \%)$, a global constant term of $0.60 \%$ is obtained. This figure is in good agreement with the expectations.

\subsection{Position and Angular Resolution}

Position resolution studies are carried out in the outer wheel of the EMEC with the same data as for the energy resolution analysis. Pseudorapidity energy-weighted barycenters are computed in the front and middle sections. The cluster size is 3 strips in the front section $(3 \times 2$ where appropriate), and $3 \times 3$ in the middle section. The same cluster size is used to compute the azimuthal barycenter in the middle section. These measurements have been compared to the beam chambers extrapolated positions, for which the precision of the track extrapolation on the calorimeter is $0.350 \mathrm{~mm}$ in both projections. Residuals distributions are constructed, and used for the finite cell size correction (S-shapes), as illustrated in Figure 29 for the front and the middle sections. Finally, the calorimeter resolution is obtained by fitting the corrected residual distributions by a Gaussian (Figure 29) and subtracting the contribution of the beam chamber prediction. Figure 30 shows the $\eta$ resolution dependence on energy at $\eta=1.9$, as well as the sampling terms for all positions. The resolution improves from $8.3 \mathrm{~mm} \mathrm{GeV}^{1 / 2}$ at $\eta=1.6$ to $3.3 \mathrm{~mm}$ $\mathrm{GeV}^{1 / 2}$ at $\eta=2.4$ in the middle section, due to the smaller cell width, while it remains constant in the front section (about $2.5 \mathrm{~mm} \mathrm{GeV}^{1 / 2}$ ), thanks to the variation of the granularity.

By combining the two pseudorapidity measurements coming from the front and the middle sections with the longitudinal shower barycenters, a measurement of the polar angle $(\theta)$ is derived. It allows to determine the primary vertex position in ATLAS for events with a $H \rightarrow \gamma \gamma$ decay at high luminosity, and to accurately reconstruct the Higgs mass. The longitudinal shower barycenters have been computed using the usual parametrisation of the longitudinal development of electromagnetic showers [26]. The polar angle resolution is the Gaussian width of the angle distribution, since the beam divergence is found to be negligible (around $0.1 \mathrm{mrad}$ ). The polar angle resolution variation with energy at $\eta=1.9$, compared to simulation, is plotted in Figure 31. The requirement on the sampling term (50 mrad $\mathrm{GeV}^{1 / 2}$ at $\left.\eta=1.9\right)$ is satisfied. The sampling term variation follows that of the middle section (Figure 31).

In the $\phi$ direction, the finite cell size correction is negligible due to the larger energy sharing between cells, a consequence of the accordion structure. The sampling term decreases from $6.5 \mathrm{~mm} \mathrm{GeV} \mathrm{V}^{1 / 2}$ at $\eta=1.6$ to $3.2 \mathrm{~mm} \mathrm{GeV}^{1 / 2}$ at $\eta=2.4$. It shows the same behaviour as the pseudorapidity sampling term in the middle section (Figure 30), due to cell size variation, but smaller, due to energy sharing.

\section{Conclusions and Prospects}

A real size electromagnetic end-cap calorimeter module, covering the region $1.375<\eta<3.2$, has been successfully constructed and tested with an ATLAS-like electronics chain. Absorber, electrode production and bending have proven to be feasible within required specifications. Using electron beam data, an energy resolution with a stochastic term between $10 \% \mathrm{GeV}^{1 / 2}$ and $12.5 \% \mathrm{GeV}^{1 / 2}$ over the full rapidity coverage is obtained, as well as position and angular measurements in agreement with the ATLAS specifications. A global constant term of $0.6 \%$ is obtained in the inner wheel. In the crack region, between inner and outer wheel, up to $60 \%$ of the energy is lost in a small zone around $\eta=2.50$. The presence of the presampler in the region $1.5<\eta<1.8$ partially compensates for the energy loss due to additional dead material located in front of the calorimeter. A fair agreement between beam test data and simulation has been found.

Before initiating module production, several changes were made in order to improve the module quality. Most of the HV problems encountered during stacking are coming from unsufficient cleanliness of the stacking room, which has been strongly improved (humidity is also regulated). On the other hand, honeycomb spacers were modified in rigid honeycomb nets, which facilitates the positioning onto the electrode (absorber). As a result, very few or no high voltage problems are detected at the end of the stacking operation for the production modules and the bulging is well under control, in the range \pm 1 $\mathrm{mm}$, without any mechanical stress. Mother and summing boards have been redesigned to reduce the high inductive crosstalk on the cold electronics by a factor of 2 , leading to an acceptable contribution. A new ground connection is also added on the electrode to obtain a better uniformity of the middle cell 
inductances in $\eta$ (Figure 8) and $\phi$.

\section{Acknowledgements}

We are indebted to our technicians for their contribution to the construction and running of the end-cap module 0 and the electronics. We would like to thank the accelerator division for the good working conditions in the H6 beam line, and Michel Lefebvre for its careful reading of this paper. Those of us from non-member states wish to thank CERN for its hospitality.

\section{References}

[1] ATLAS Collaboration, Technical Proposal for a General Purpose pp Experiment at the Large Hadron Collider at CERN, CERN/LHCC/94-43.

[2] ATLAS Collaboration, Liquid Argon Calorimeter Technical Design Report, CERN/LHCC/96-41.

[3] ATLAS Collaboration, Calorimeter Performance Technical Design Report, CERN/LHCC/96-40.

[4] RD3 Collaboration, Nucl. Instrum. Methods A 389 (1997) 398.

[5] S. Klimenko, Yu. Tikhonov and A. Chekhtman, The design of endcap EM calorimeter with constant thickness of the absorber plates, ATL-LARG-025 (1995).

[6] G. Garcia et al., Nucl. Instrum. Methods A 418 (1998) 513.

[7] F. Djama, L. Hervas and C.P. Marin, Copper-Polyimide Multi-Layer Electrodes for the End-Cap Electromagnetic Calorimeter, ATL-LARG-98-088.

[8] F. Astesan et al., Description and Performances of the Electrical Test Benches for Readout Electrodes of the ATLAS EM Calorimeter, ATL-LARG-99-005.

[9] W. Bonivento, Impact of resistor quality of the readout electrodes on e.m. calorimeter performance, ATL-LARG-97-081.

[10] W. Bonivento and D. Lacour, Acceptable Values of Resistances on Electrodes of the ATLAS e.m. Calorimeter, ATL-LARG-99-019.

[11] P. Dargent and F. Djama, Bending of End-Cap Electrodes, ATL-LARG-98-108.

[12] H. Burkhardt et al., Nucl. Instrum. Methods A 268 (1988) 116.

[13] M. Chalifour et al., Cryogenic System for the Test Facilities of the ATLAS Liquid Argon Calorimeter Modules, CERN-LHC-Project-Report-234.

[14] J. Spanggaard, Delay Wire Chambers - A Users Guide, SL-Note-98-023 BI.

[15] ATLAS Electromagnetic Liquid Argon Calorimeter Group, Performance of the ATLAS Electromagnetic Calorimeter Barrel Module 0, CERN-EP/2002-087, accepted for publication in Nucl. Instrum. Methods A.

[16] C.P. Bee et al., A scalable data taking system at a test beam for LHC, CERN/LHCC/95-47.

[17] W. E. Cleland and E. G. Stern, Nucl. Instrum. Methods A 338 (1994) 467.

[18] P. Barrillon et al., Signal Reconstruction in Module 0 of the End-Cap Electromagnetic Calorimeter and Presampler, ATL-LARG-2001-022.

[19] B. Mansoulié and J. Schwindling, Using Multi Layer Perceptrons in PAW, http://schwind.home.cern.ch/schwind/MLPfit.html.

[20] P. Pralavorio and D. Sauvage, Review of the crosstalk in the module 0 of the Electromagnetic Endcap Calorimeter, ATL-LARG-2001-006.

[21] J. Colas et al., Crosstalk in the ATLAS Electromagnetic Calorimeter, ATL-LARG-2000-004.

[22] P. Barrillon et al., Uniformity Scan in the Outer Wheel of the Electromagnetic Endcap Calorimeter, ATL-LARG-2001-012.

[23] G. Garcia, PhD. Thesis, DESY-THESIS-2000-010.

[24] V.M. Aulchenko et al., Performance of the EM end-cap presampler in the 1999 testbeam run, ATLLARG-2001-016.

[25] P. Barrillon et al., Uniformity scan in the Inner Wheel and the crack between the two wheels (eta=2.5) of the Electromagnetic End-cap Calorimeter, ATL-LARG-2001-014.

[26] E. Longo and I. Sestili, Nucl. Instrum. Methods A 128 (1975) 283. 
Table 1: Granularity $(\Delta \eta \times \Delta \phi)$ of calorimeter cells in the outer and inner wheel of the EMEC.

\begin{tabular}{|l|l||c|c|c|}
\hline Wheel & $\eta$ range & Front & Middle & Back \\
\hline Outer & {$[1.375,1.425]$} & $0.050 \times 0.1$ & $0.050 \times 0.025$ & - \\
& {$[1.425,1.5]$} & $0.025 \times 0.1$ & $0.025 \times 0.025$ & - \\
& {$[1.5,1.8]$} & $\sim 0.003 \times 0.1$ & $0.025 \times 0.025$ & $0.050 \times 0.025$ \\
& {$[1.8,2.0]$} & $\sim 0.004 \times 0.1$ & $0.025 \times 0.025$ & $0.050 \times 0.025$ \\
& {$[2.0,2.4]$} & $\sim 0.006 \times 0.1$ & $0.025 \times 0.025$ & $0.050 \times 0.025$ \\
& {$[2.4,2.5]$} & $0.025 \times 0.1$ & $0.025 \times 0.025$ & $0.050 \times 0.025$ \\
\hline Inner & {$[2.5,3.2]$} & - & $0.1 \times 0.1$ & $0.1 \times 0.1$ \\
\hline
\end{tabular}

Table 2: Cluster size $\left(\Delta \eta_{\text {cell }} \times \Delta \phi_{\text {cell }}\right)$ per layer around cell with maximum energy deposit, ${ }^{*}$ only for $E_{\text {beam }}>50 \mathrm{GeV}$.

\begin{tabular}{|l||c|c|c|c||c|}
\hline \multicolumn{1}{|l||}{$\begin{array}{l}\text { Wheel } \\
\eta \text {-range }\end{array}$} & \multicolumn{4}{|c||}{ Outer } & Inner \\
\cline { 2 - 6 } & {$[1.5,1.8]$} & {$[1.8,2.0]$} & {$[2.0,2.4]$} & {$[2.4,2.5]$} & {$[2.5,3.2]$} \\
\hline PS & $3 \times 1(2)$ & - & - & - & - \\
\hline Front & $23 \times 1(2)$ & $15 \times 1(2)$ & $11 \times 1(2)$ & $3 \times 1(2)$ & - \\
\hline Middle & $3 \times 3,5 \times 5$ & $5 \times 5$ & $5 \times 5$ & $5 \times 5$ & $3 \times 3$ \\
\hline Back & $1 \times 1^{*}$ & $1 \times 1^{*}$ & $1 \times 1^{*}$ & $1 \times 1^{*}$ & $1 \times 1^{*}$ \\
\hline
\end{tabular}




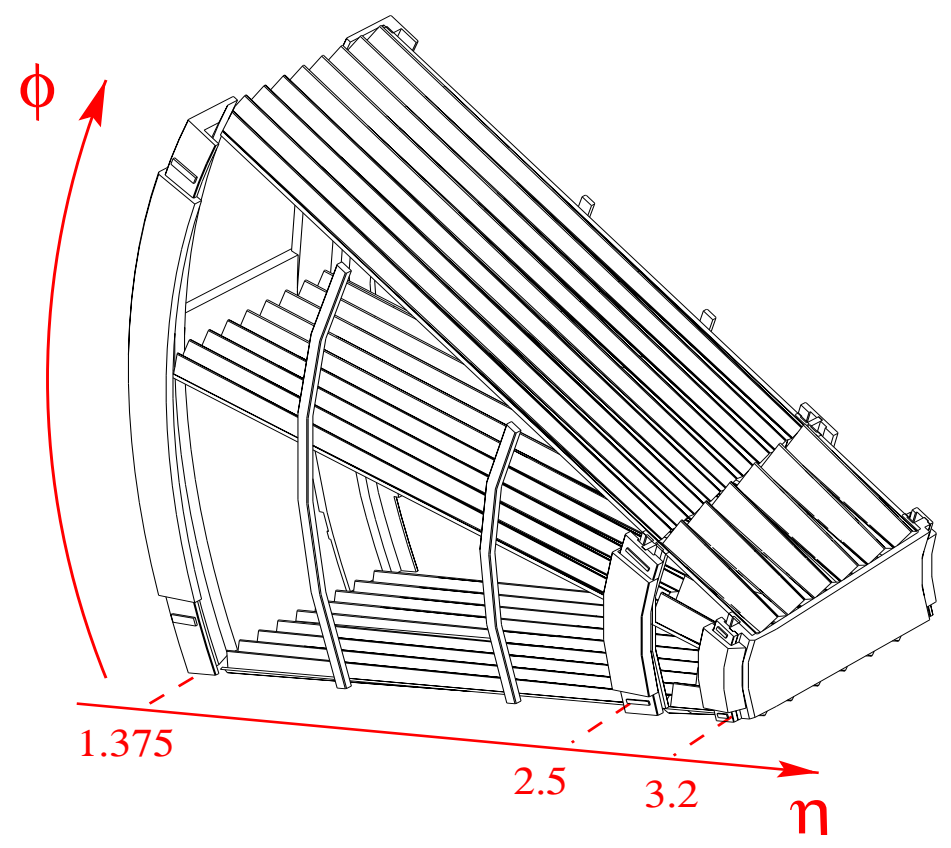

Figure 1: Schematic view of an electromagnetic end-cap module (the anti-sagitta ring at the outer radius is not shown).

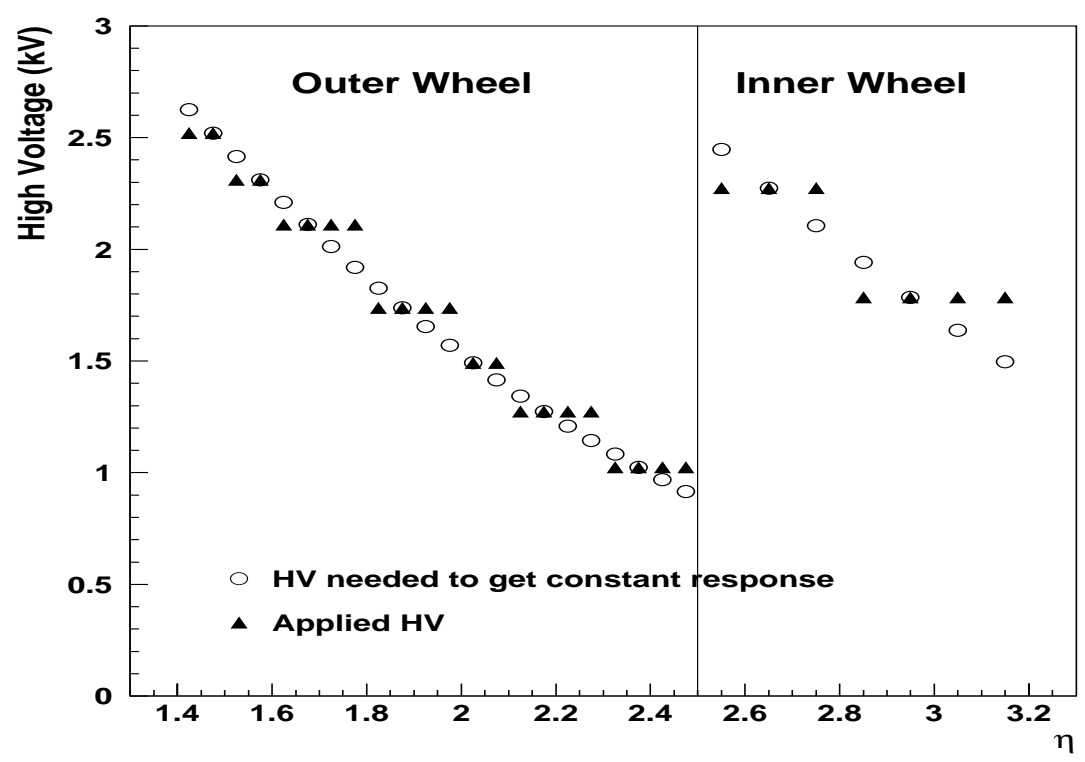

Figure 2: High voltage variation along $\eta$ for a uniform calorimeter response (open circles). The chosen high voltage settings (in liquid argon) are shown with solid triangles. 

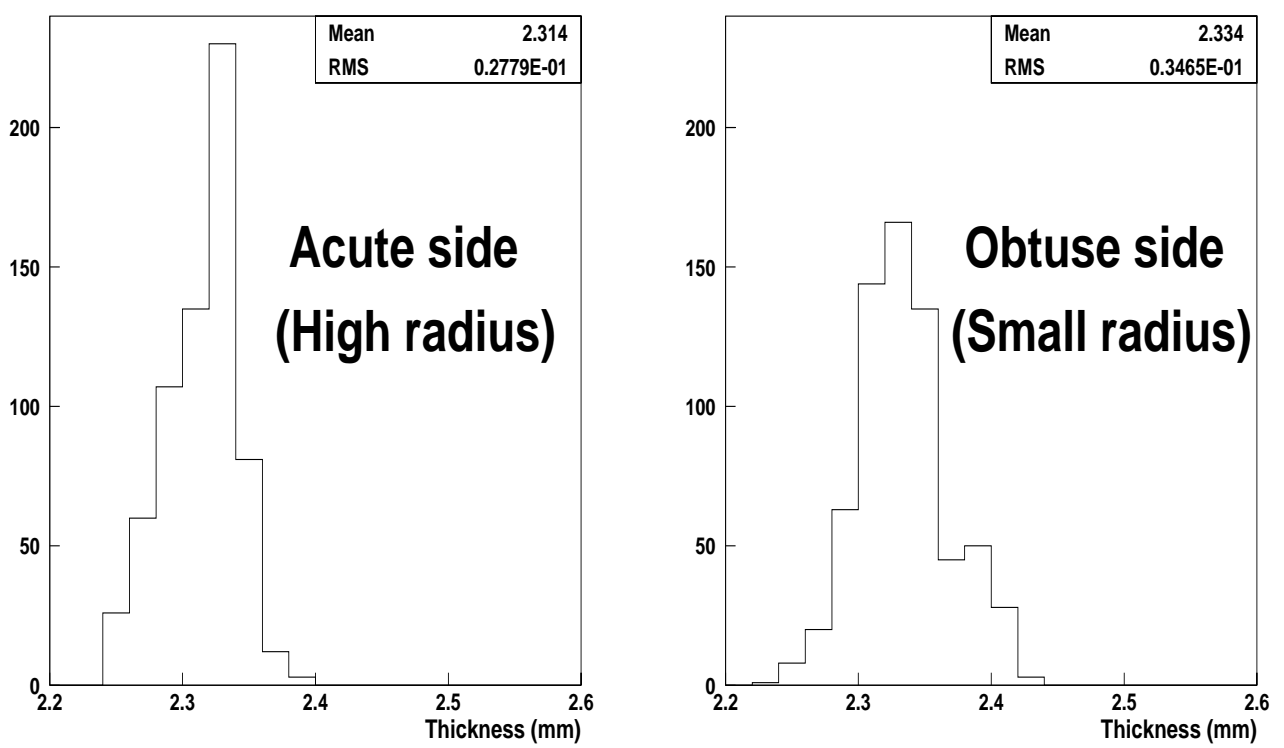

Figure 3: Thickness measurement at both edges of the absorbers in the outer wheel.

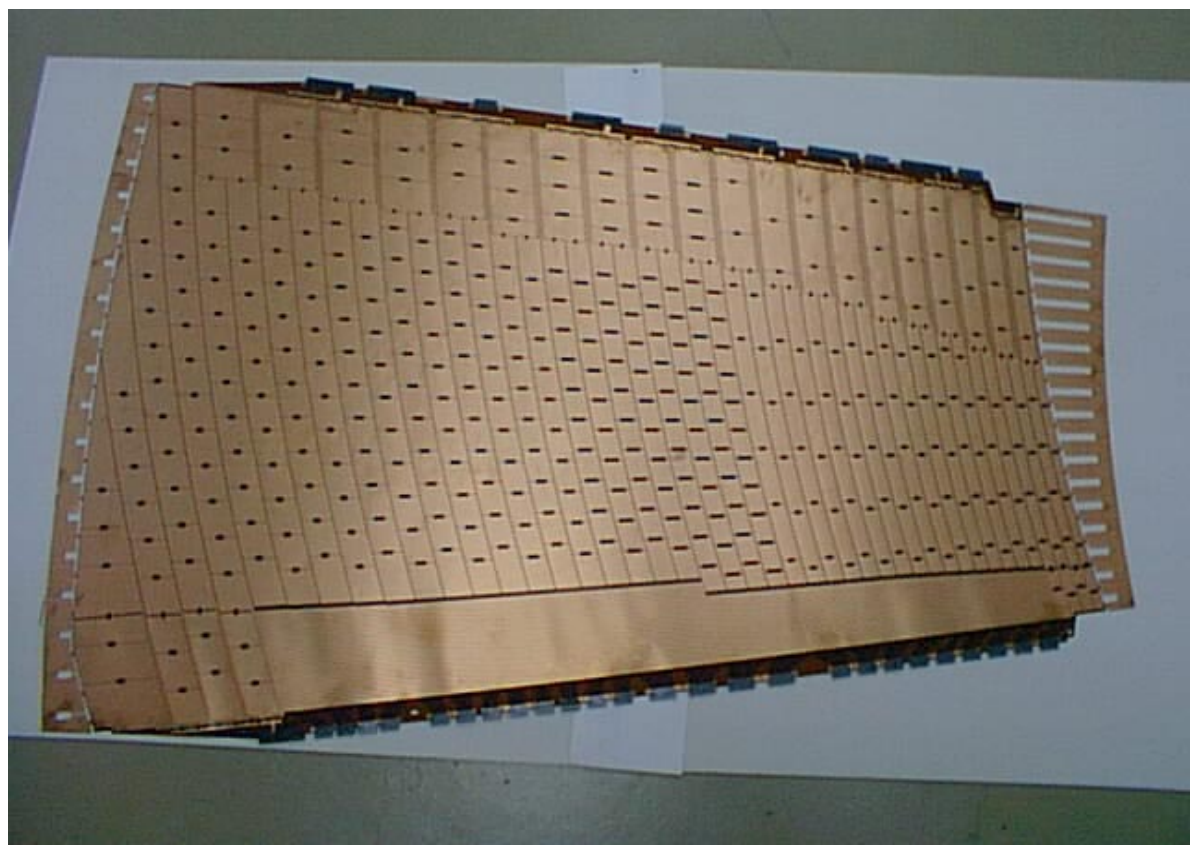

Figure 4: Picture of an unbent outer wheel electrode. The electrode size is $956 \mathrm{~mm}$ large and $1445 \mathrm{~mm}$ long. 


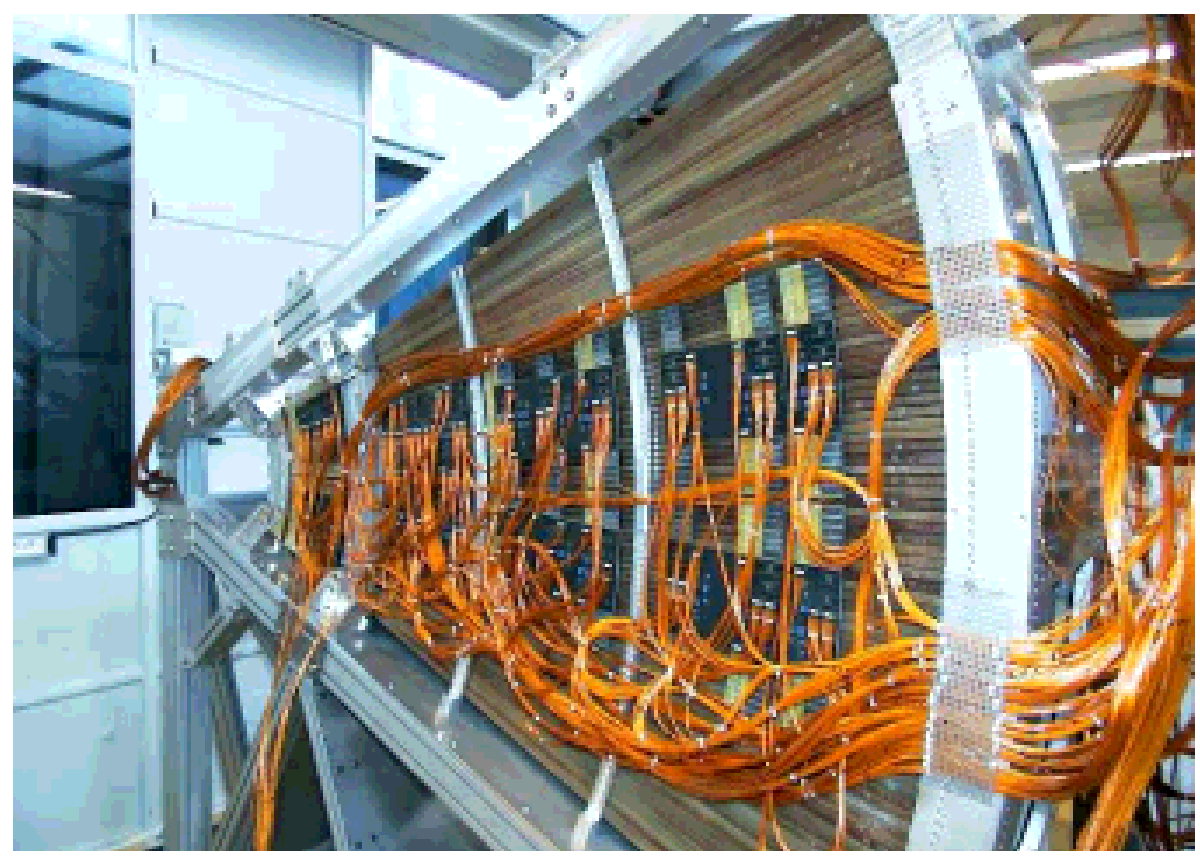

Figure 5: Back side of the module 0 after cabling. Support structure and intermediate rings are shown in this picture.

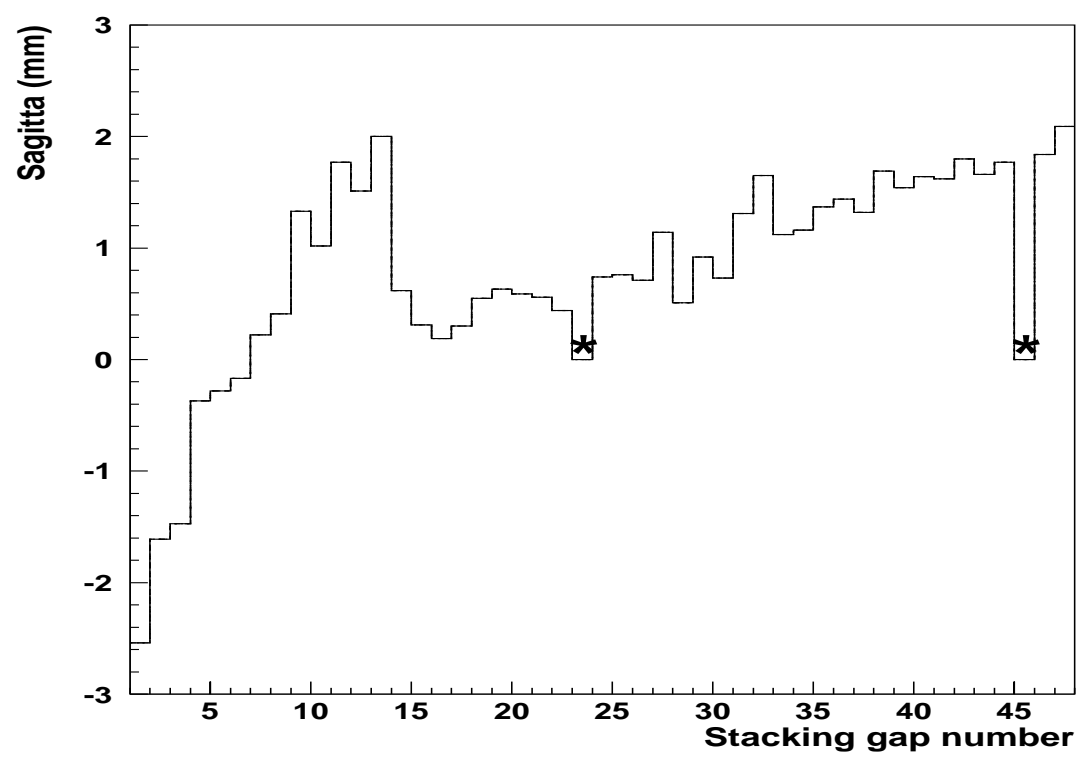

Figure 6: Sagitta measurements (performed on the last mounted absorber) in the central wave at $\eta \sim 1.8$ in the outer wheel. The stars indicate a missing measurement, put to 0. 


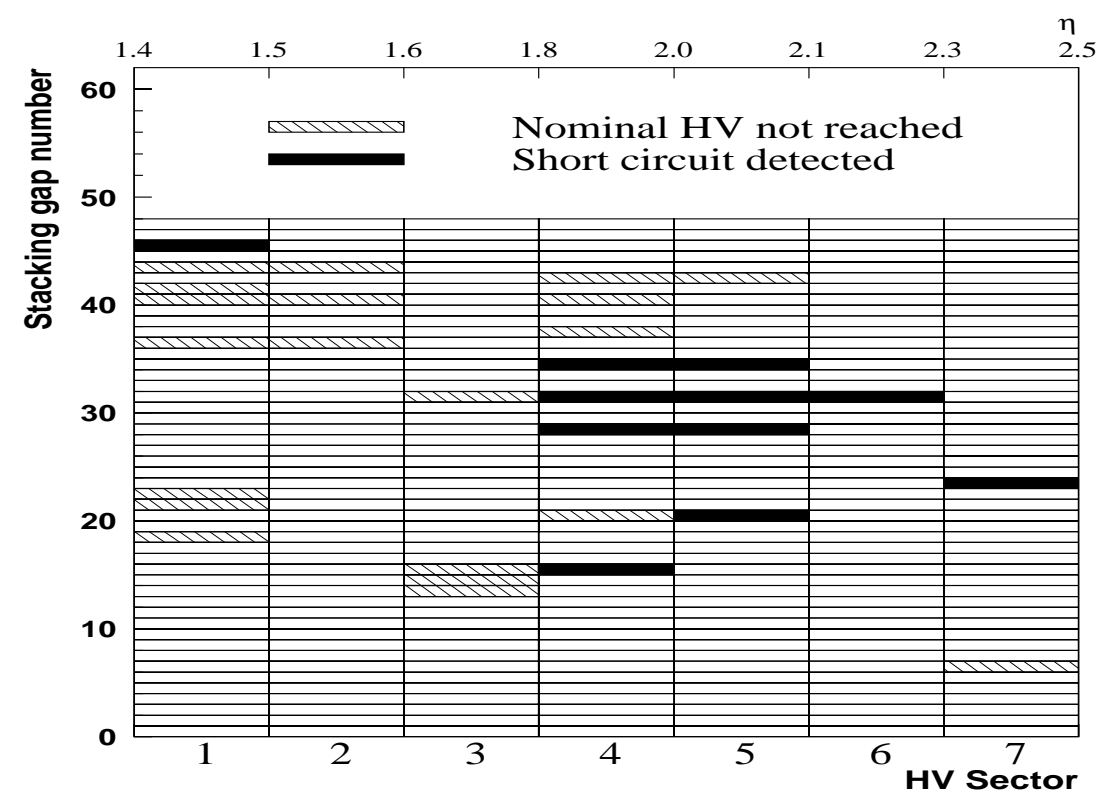

Figure 7: Map of $H V$ problems encountered on a stacking gap after module completion. For each problem, only one half of the gap is concerned.
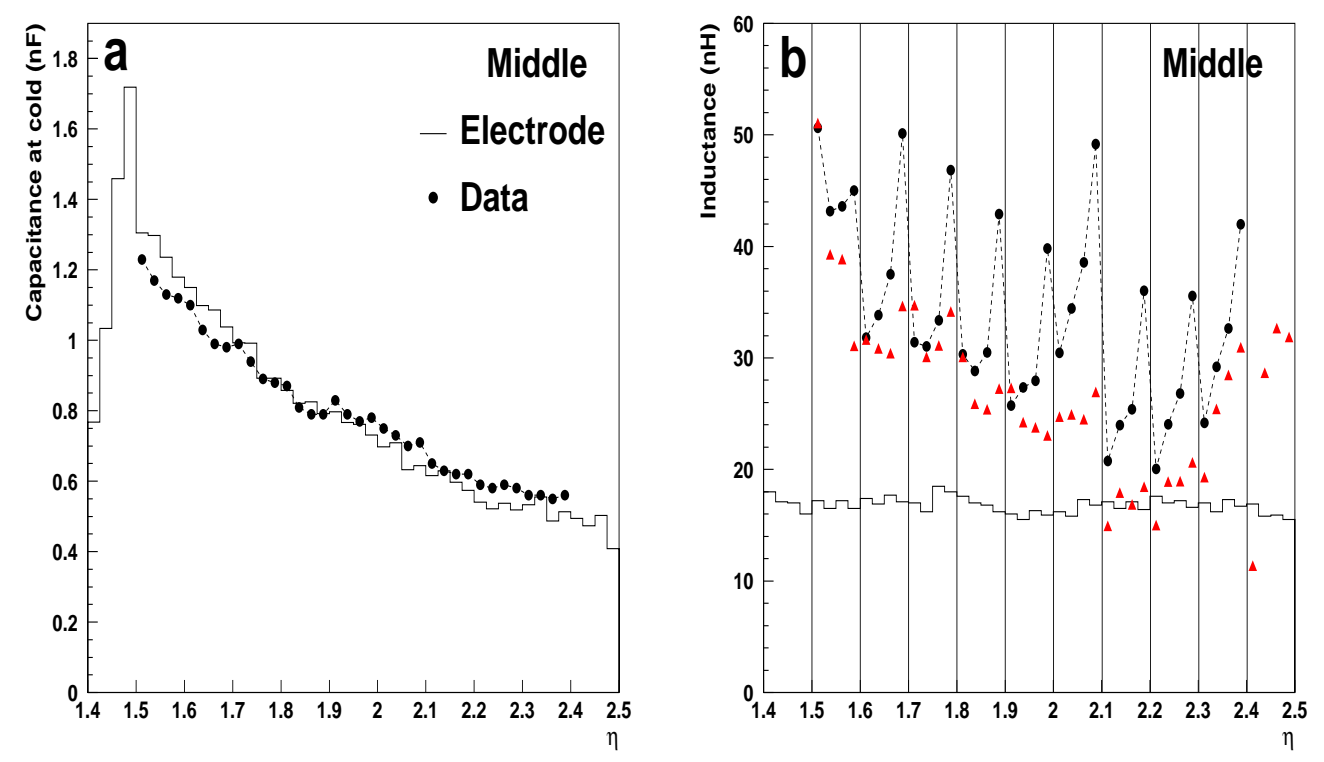

Figure 8: a) Capacitances at liquid argon temperature and b) inductances measured in the middle section with an RLC meter at the summing board level (solid points), for a given $\phi$ of the module 0. The solid histograms show the theoretical capacitances and inductances [7] coming from the electrode. On b), vertical lines represent the separation between summing boards, and triangles are measurement of the first production module (see section 8). 

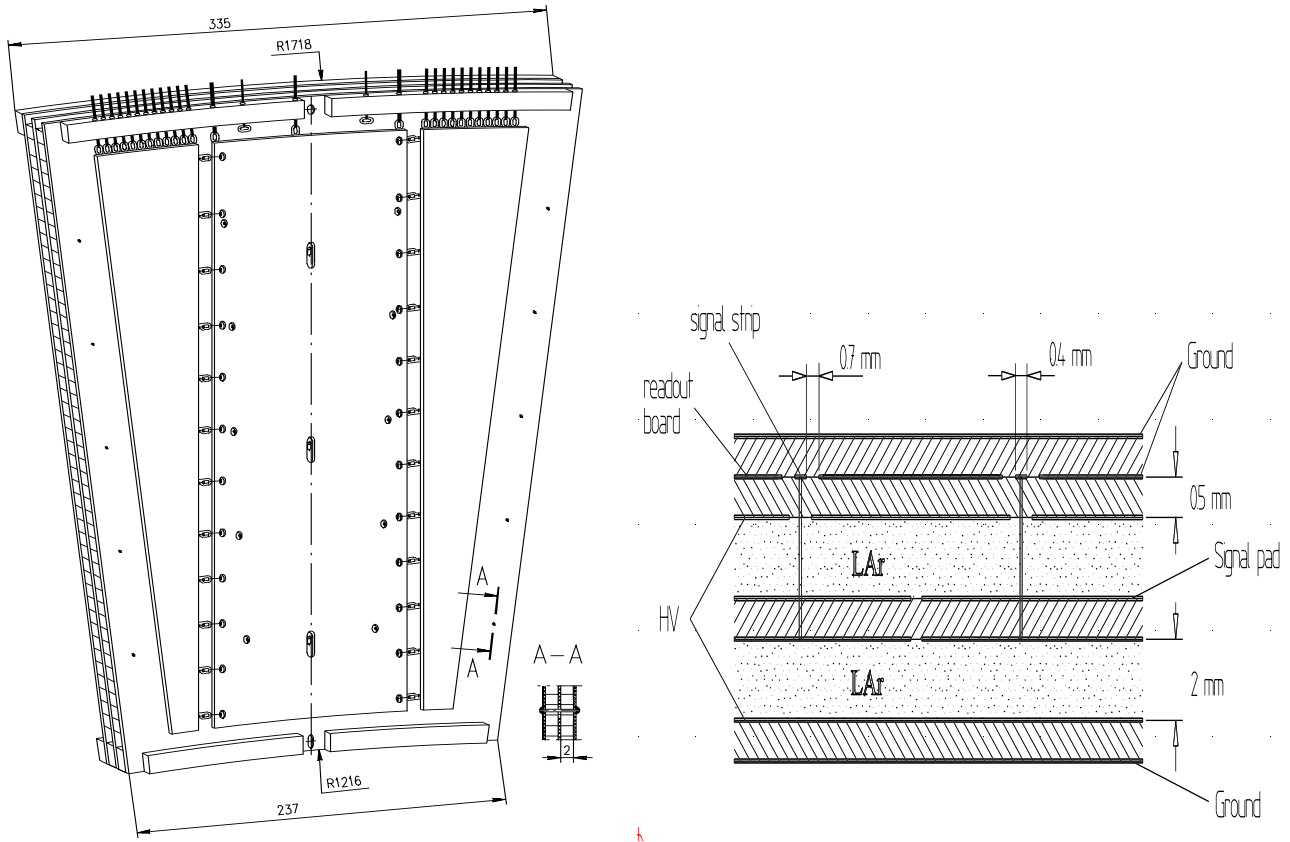

Figure 9: Isometric view of a presampler module (left) and presampler design principle (right). Units are in $\mathrm{mm}$. 


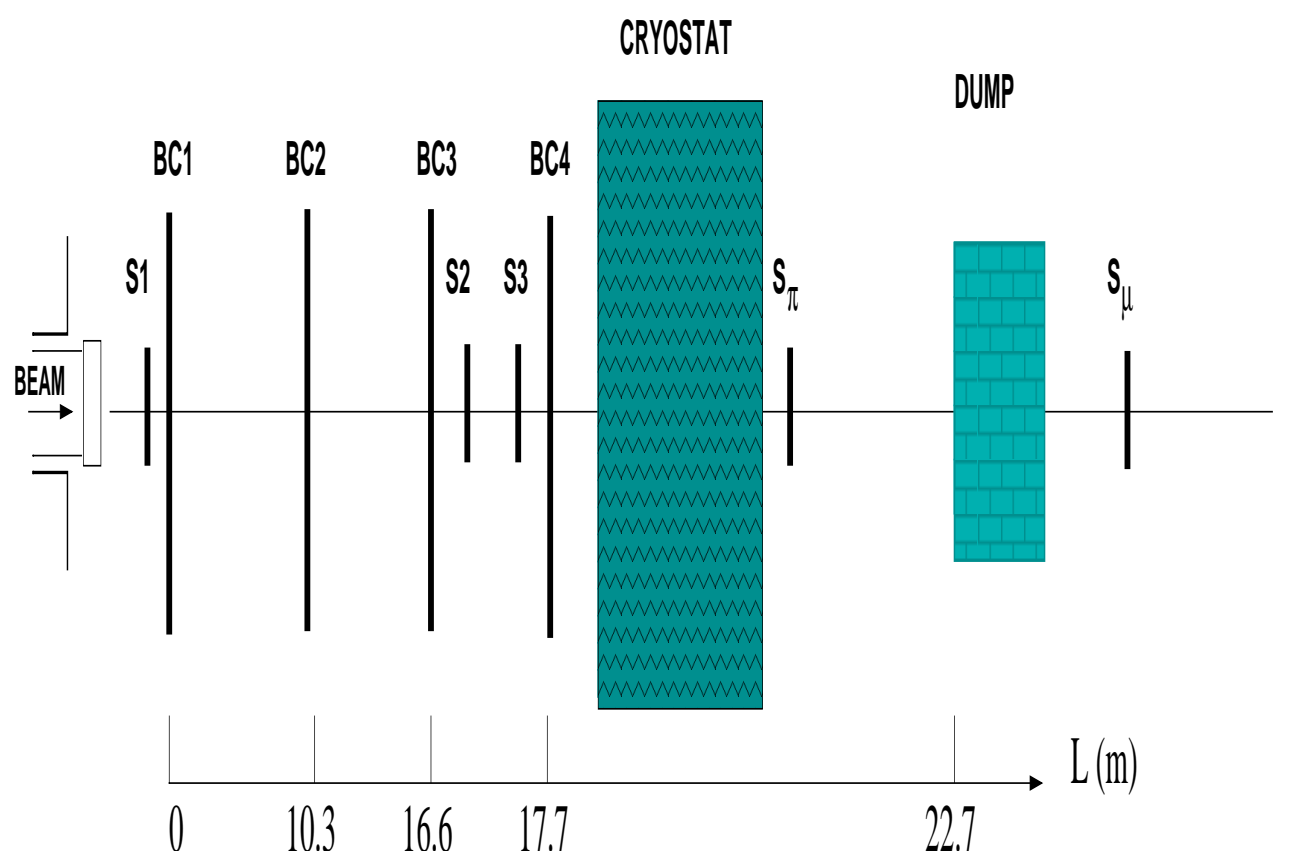

Figure 10: Schematic view of the H6 beam line instrumentation. Scintillators are labelled $S$ and beam chambers $B C$. 

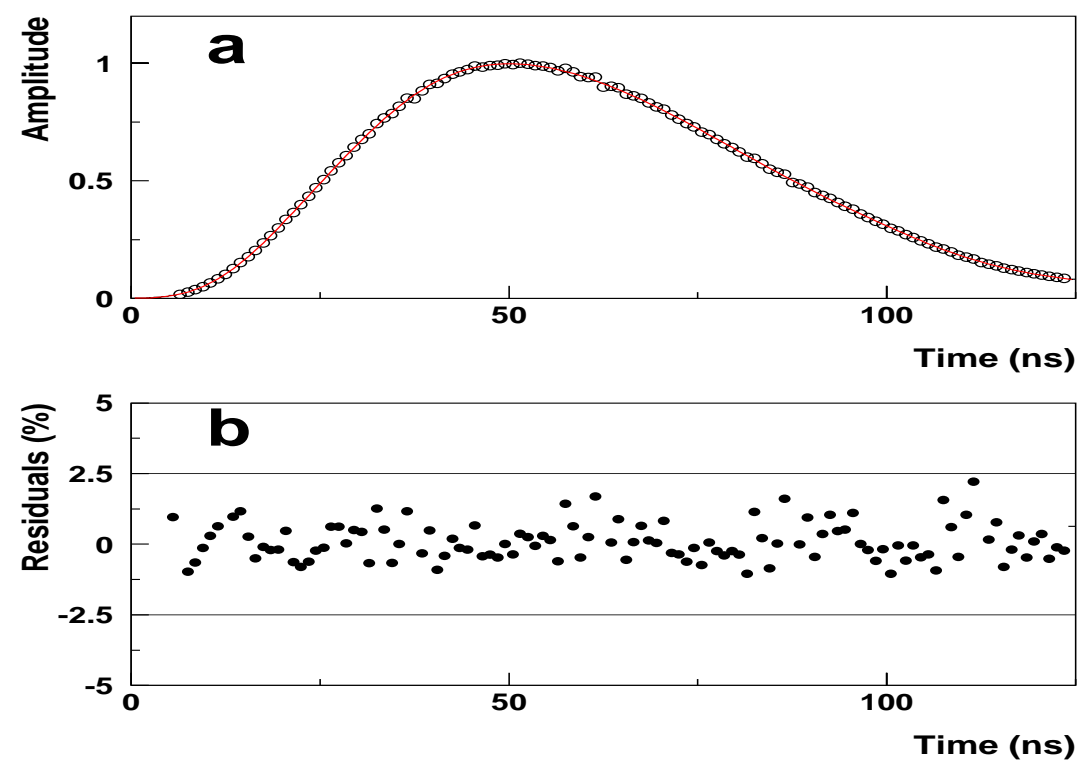

Figure 11: Reconstructed physics shape of a middle cell in medium gain, its fit with sigmoid sum function (a), and residuals of the fit (b).
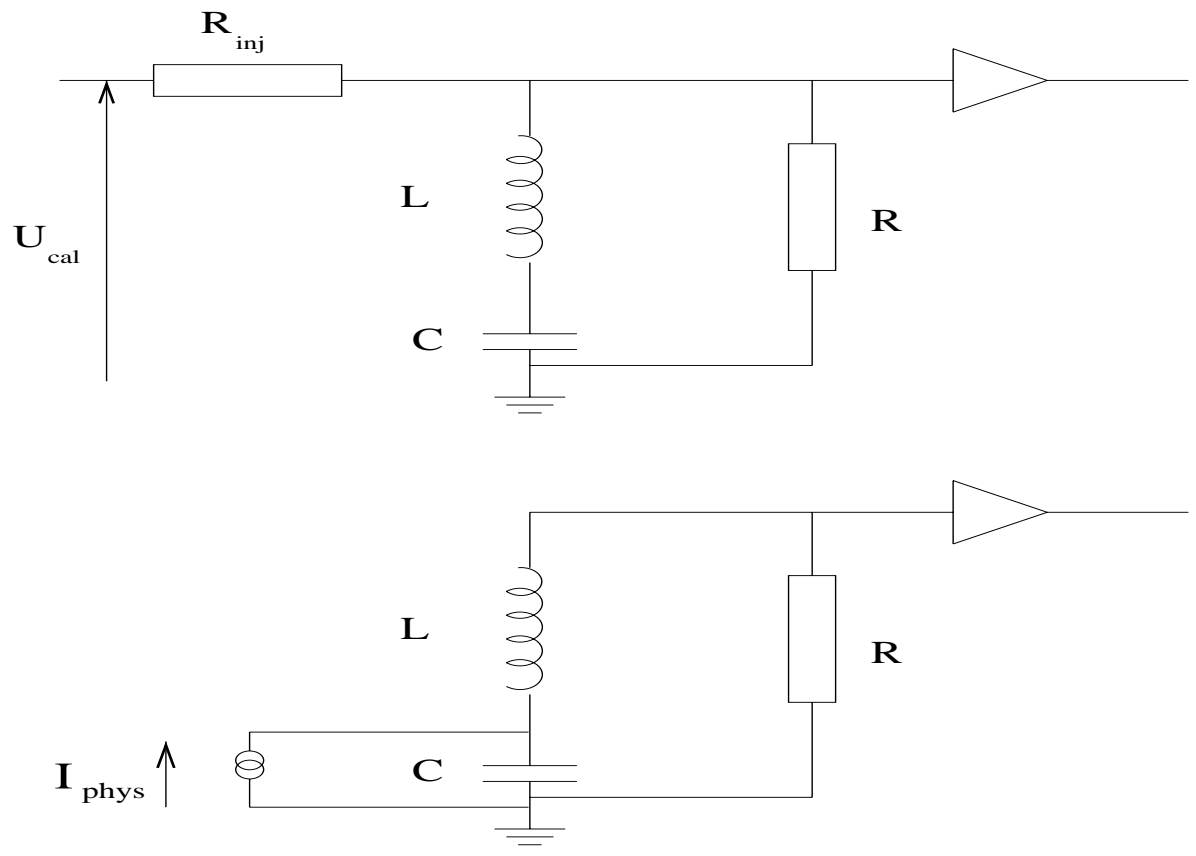

Figure 12: Electrical description of calibration (top) and physics (bottom) signal for one calorimeter cell. $R_{\text {inj }}$ are the injection resistors for calibration, $C$ and $L$ the cell capacitance and inductance of the cell, and $R$ the characteristic impedance of the readout cable. (50 $\Omega$ for presampler and front section, $25 \Omega$ elsewhere.) 


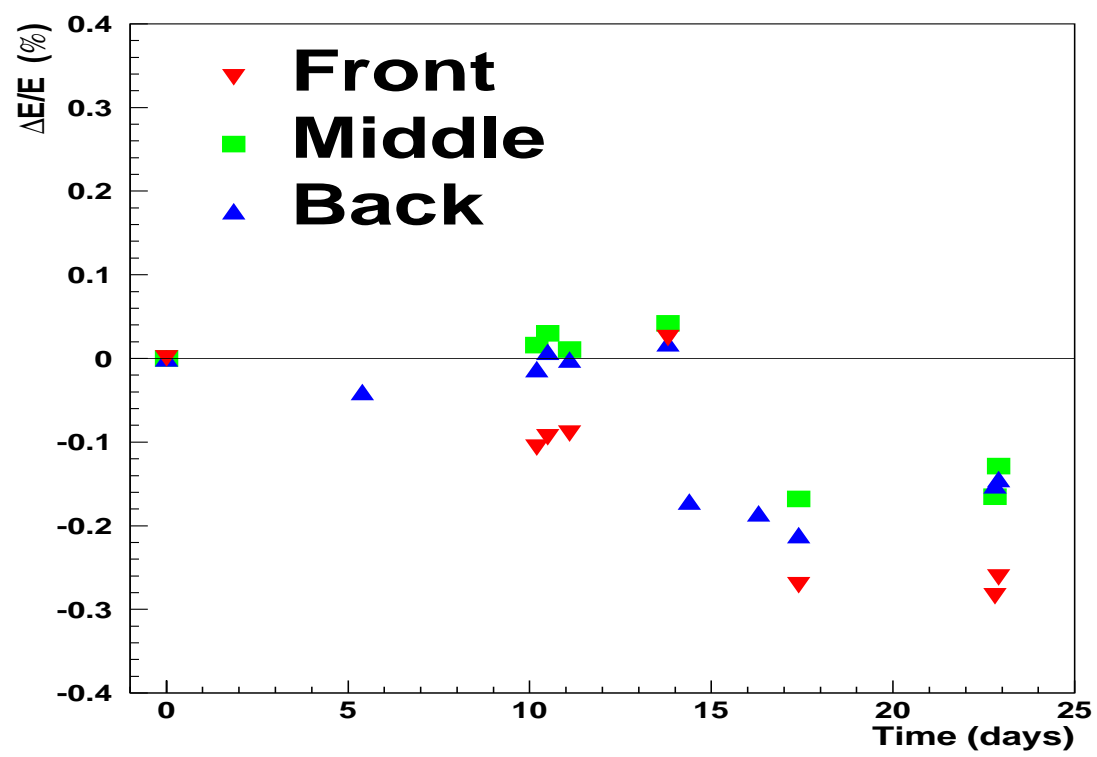

Figure 13: Calibration variation with time in the three compartments of the calorimeter.

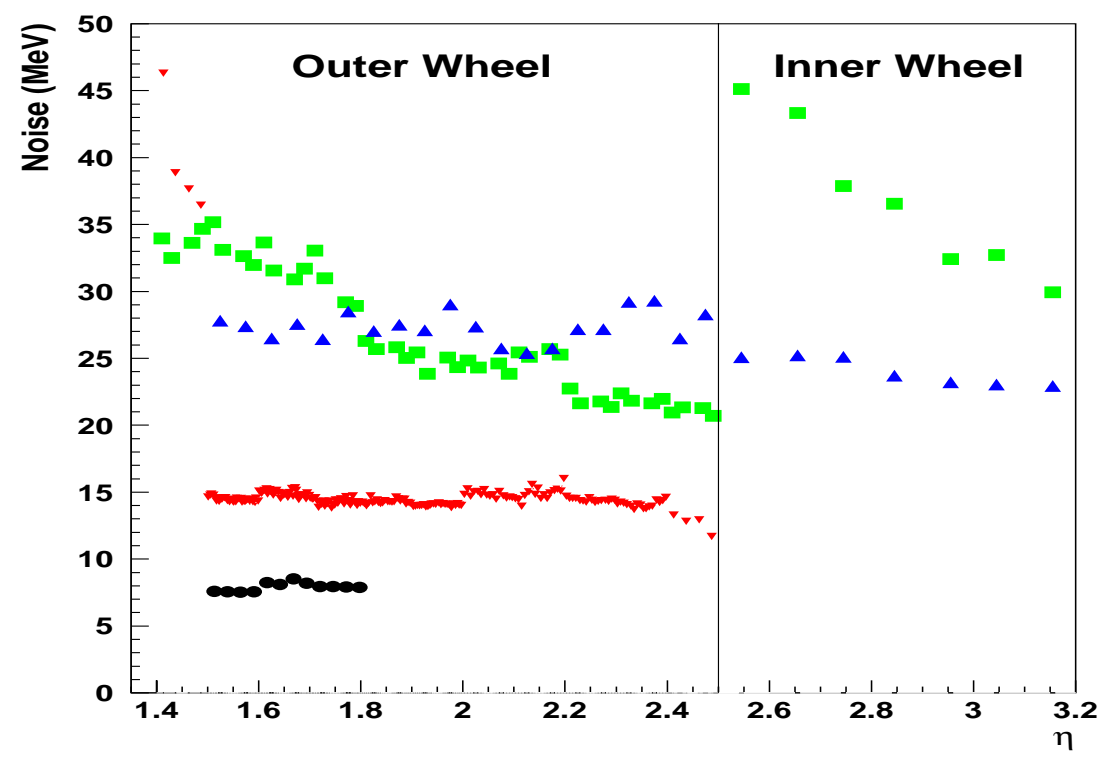

Figure 14: Total noise in high gain per readout cell (averaged in $\phi$ ), as a function of $\eta$, for the presampler (solid circles) the front (down triangles), middle (squares), back (up triangles) sections. The presampler noise does not include the relative weight with respect to the calorimeter. 


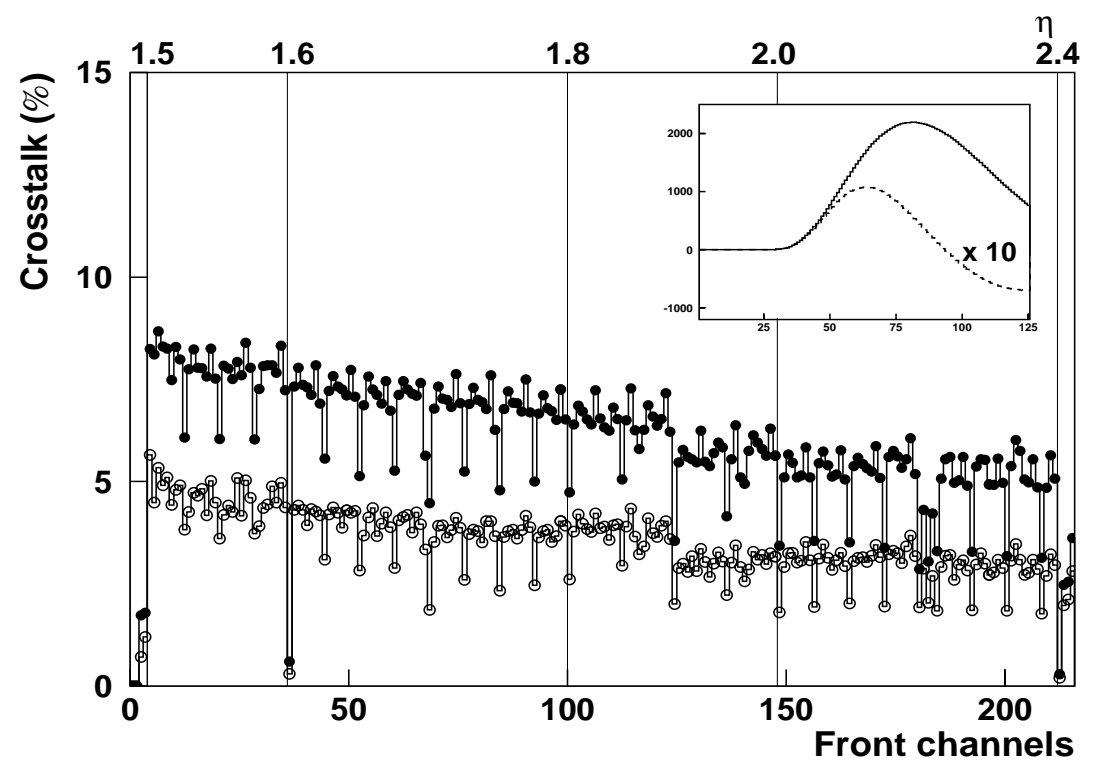

Figure 15: Crosstalk in the front section. Solid (open) circles represent the crosstalk peak-to-peak (at the signal peak). A typical signal (crosstalk) shape is shown with solid (dashed) line in the encapsulated picture. The crosstalk shape is multiplied by 10.

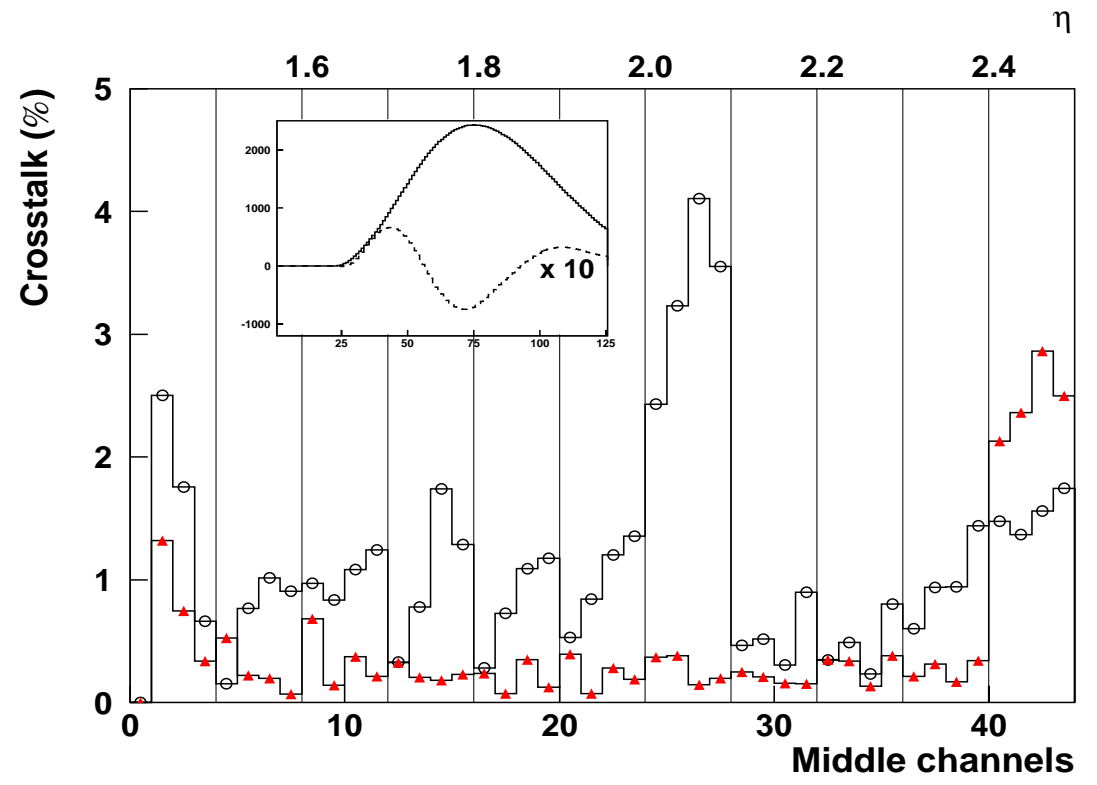

Figure 16: Crosstalk under the peak in the middle section. Open circles represent the crosstalk amplitude for module 0, and triangles for the first production module. Vertical lines represent the separation between the summing boards. A typical signal (crosstalk) shape of Module 0 is shown with solid (dashed) line in the encapsulated picture. The crosstalk shape is multiplied by 10. 


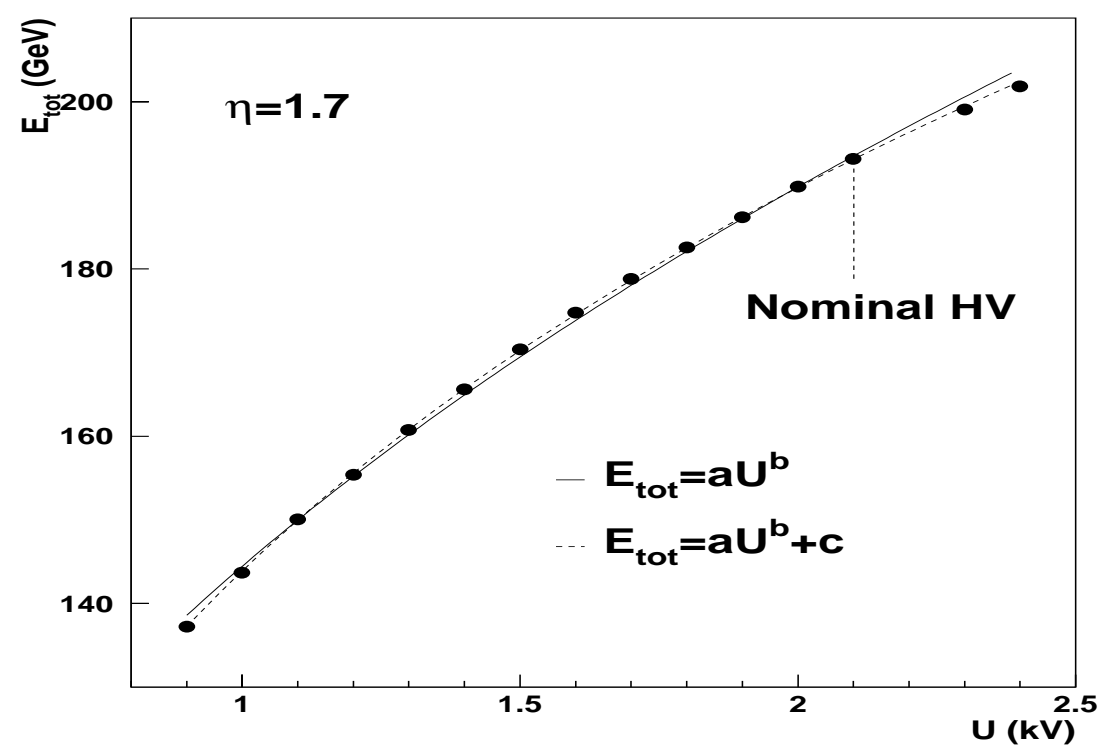

Figure 17: $H V$ dependence of the energy at $\eta=1.7$ obtained with a 193 GeV electron beam. A value of $b=0.39$ (0.12) is obtained from the fit with 2 (3) parameters.

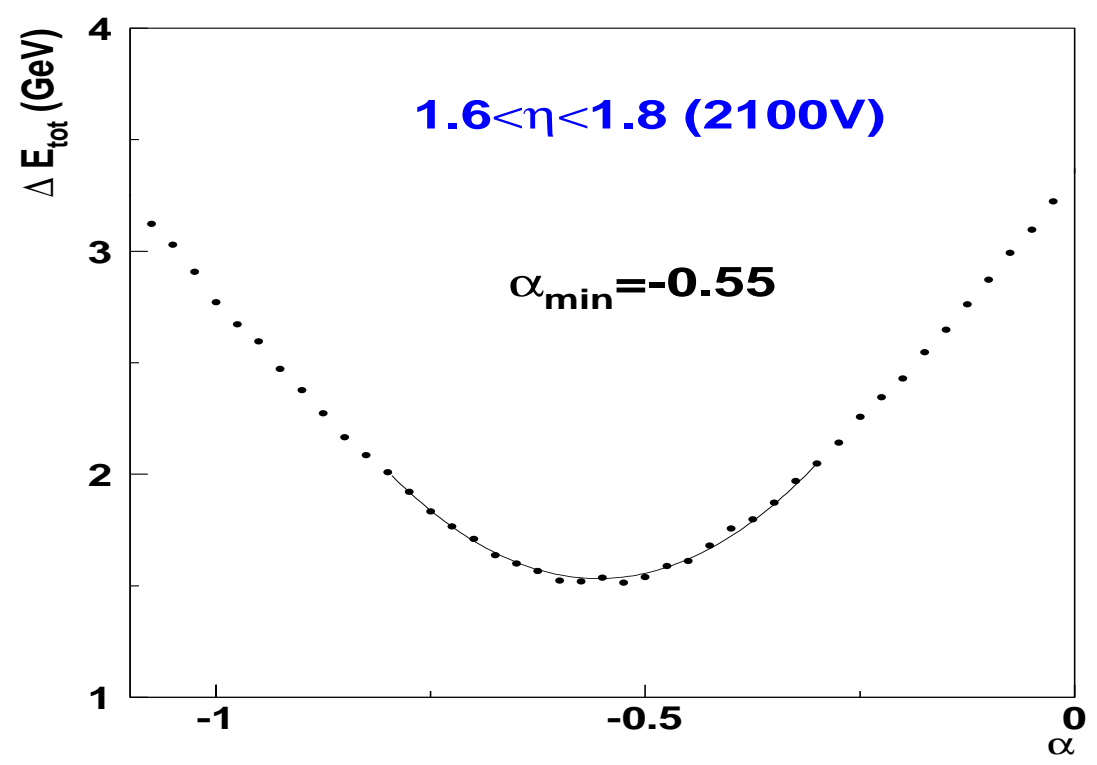

Figure 18: Variation of the dispersion of the total energy, computed for each middle cells, in the region $1.6<\eta<1.8$ (8 middle cells) for different $\alpha$ parameters. The curve is a parabola fit. 

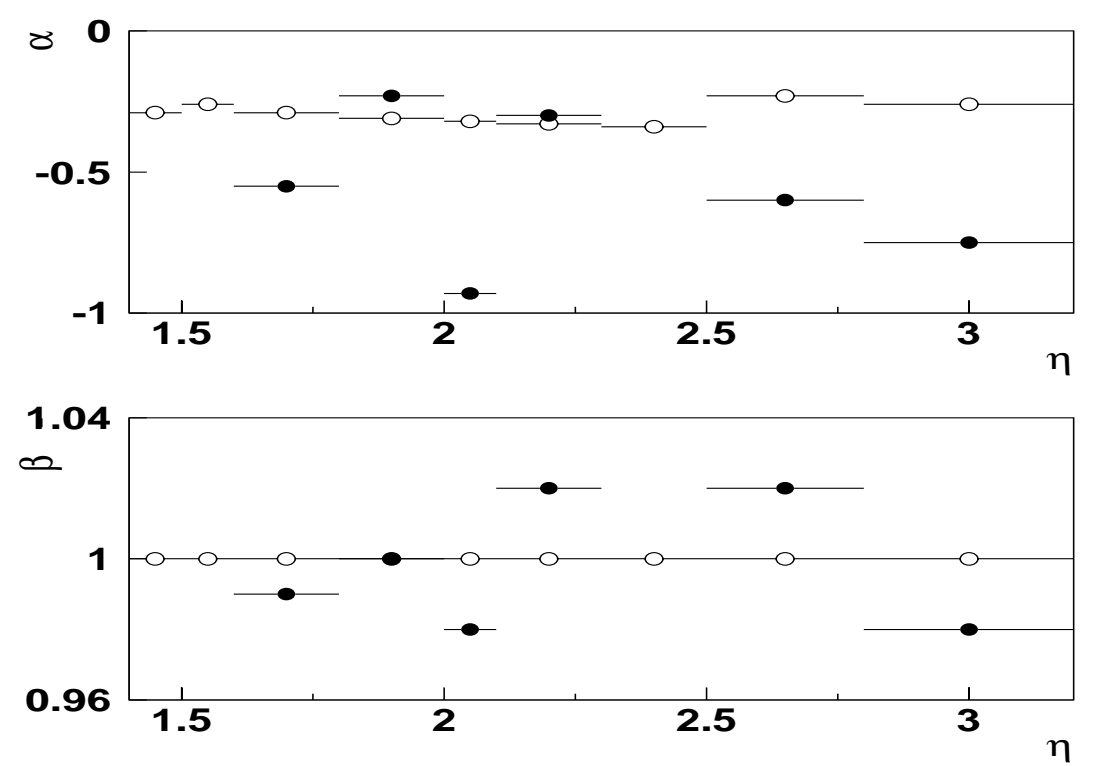

Figure 19: $\alpha$ and $\beta$ parameters values (one value per high voltage sector) as a function of $\eta$ for beam test data (solid circles) and simulation (open circles).
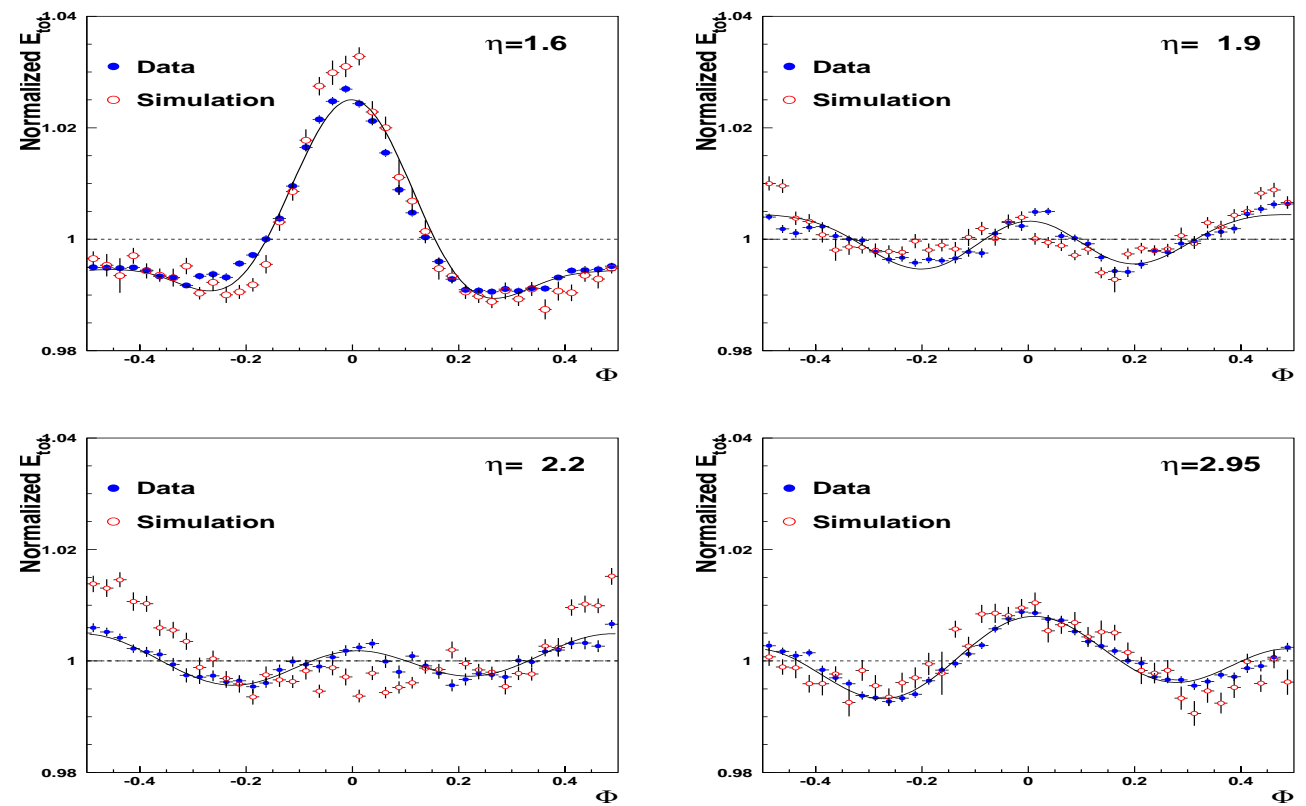

Figure 20: $\phi$-modulations in absorber units at different $\eta$ for a $100 \mathrm{GeV}$ beam. Beam test data are shown with solid circles and simulation results with open ones. The result of a Fourier series fit is shown for the beam test data. 

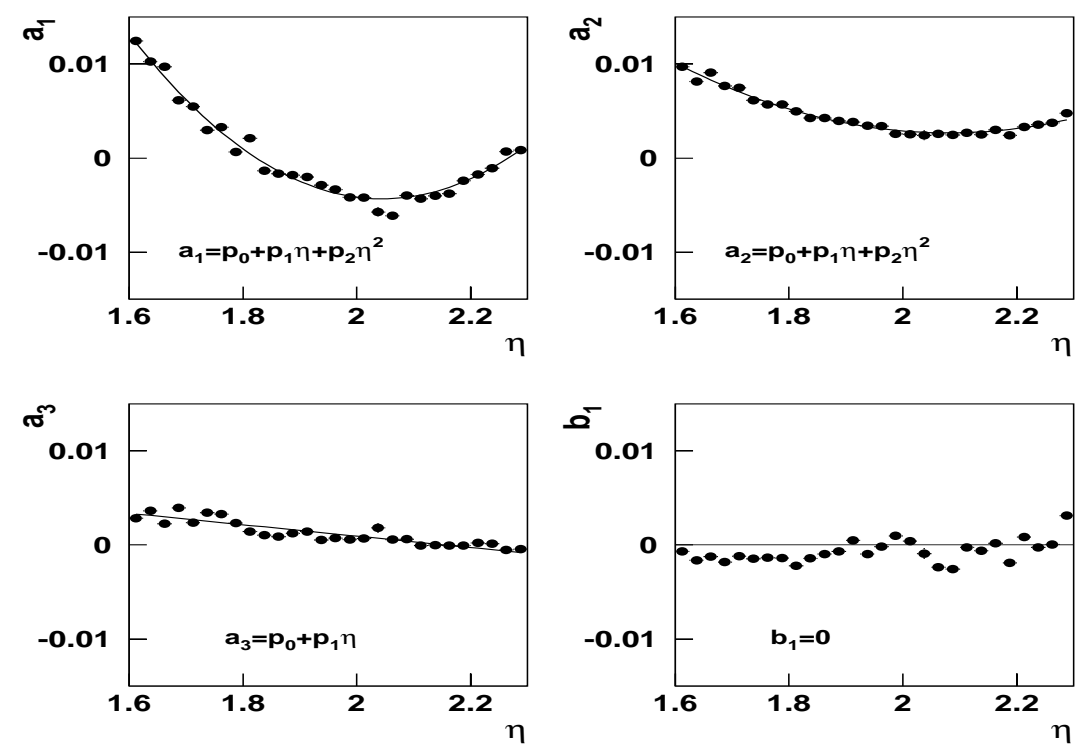

Figure 21: Parameters of $\phi$-modulation as a function of $\eta$ for a 100 GeV beam, in the outer wheel.

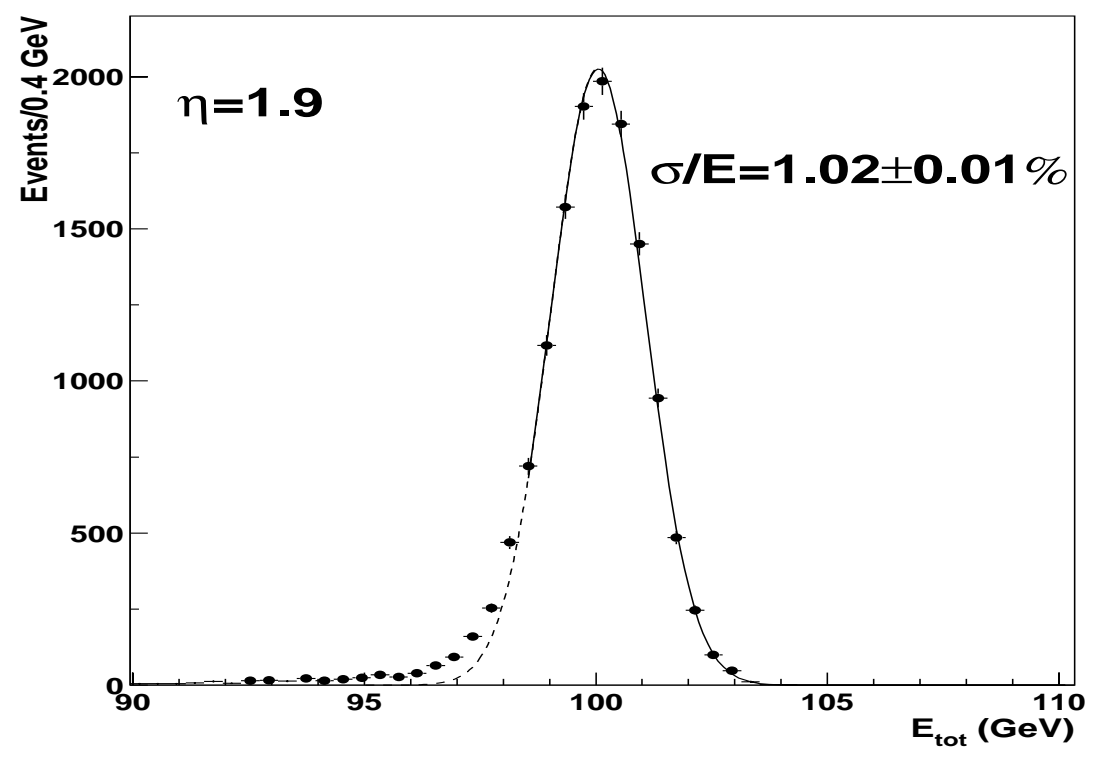

Figure 22: Total energy distribution at $\eta=1.9$ after all corrections. The Gaussian fit is for $E_{\text {tot }}>100-1.5 \sigma$ GeV. 

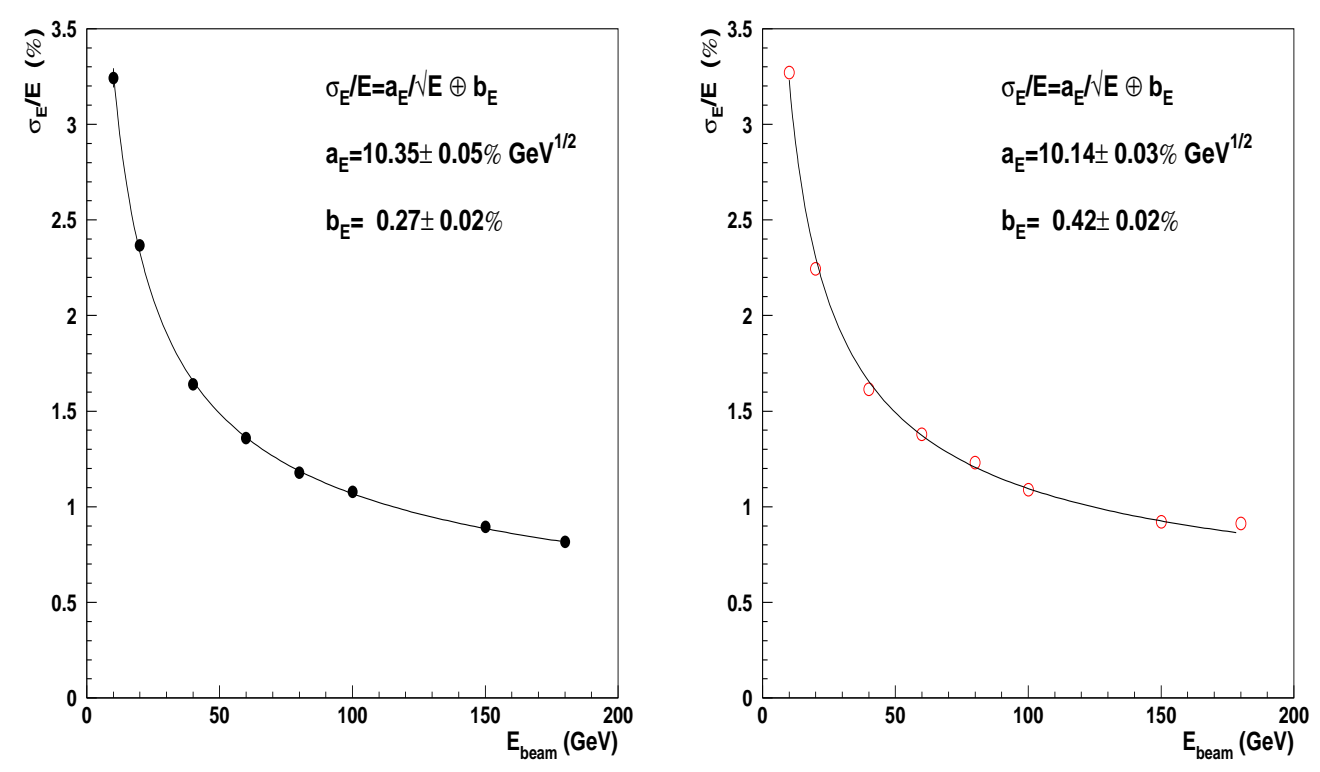

Figure 23: Energy Resolution at $\eta=1.9$ for beam test data (left) and simulation (right).
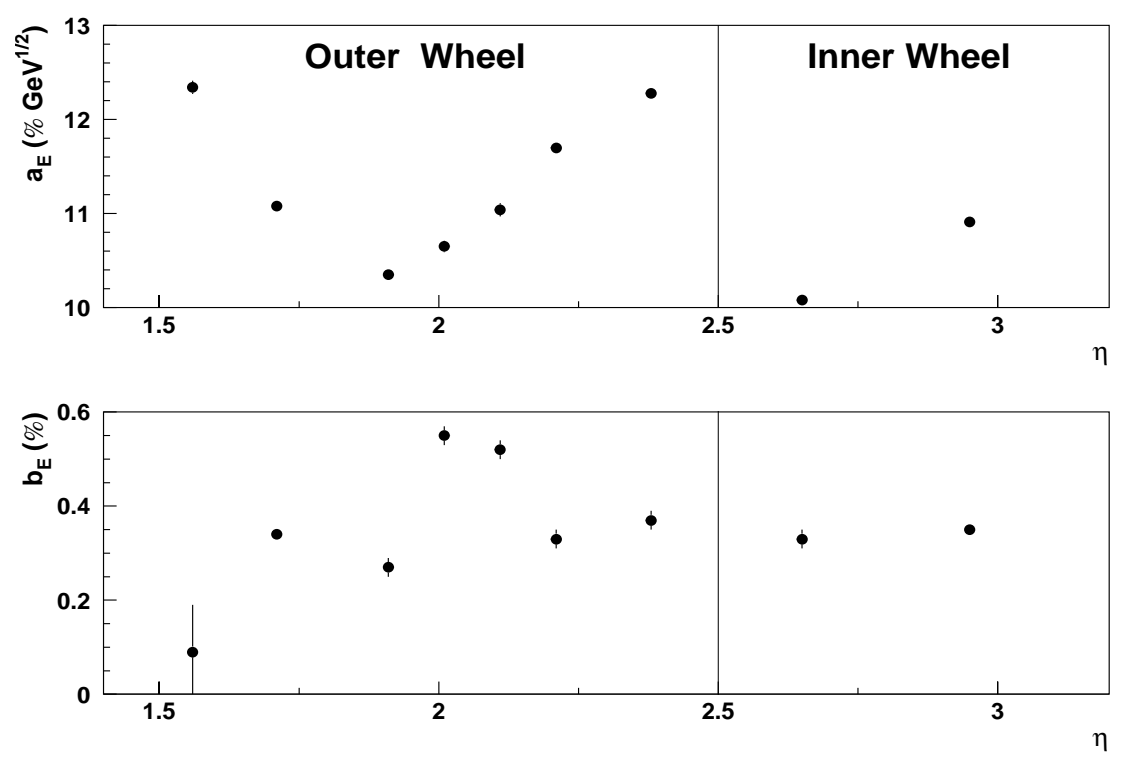

Figure 24: Variation of the stochastic term $\left(a_{E}\right)$ and the local constant term $\left(b_{E}\right)$ as a function of $\eta$. 

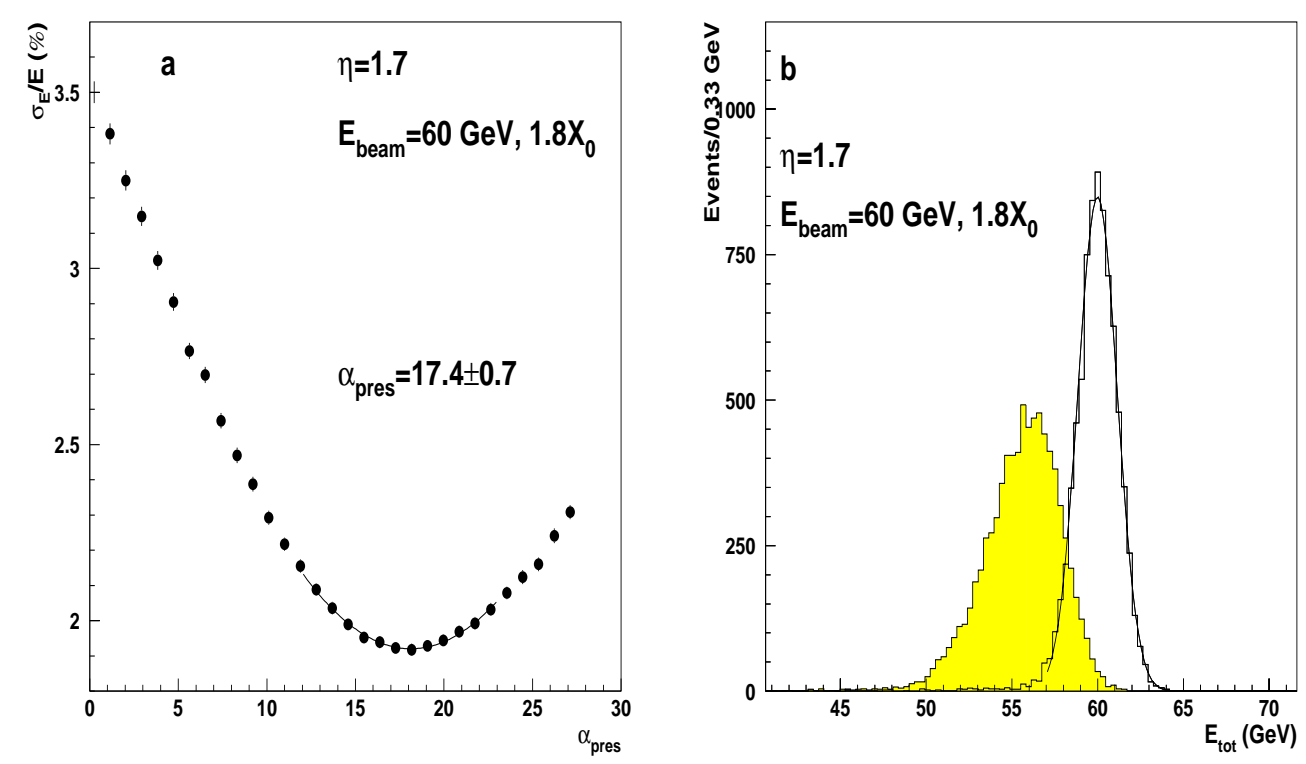

Figure 25: a) Energy resolution as a function of the presampler weight at $60 \mathrm{GeV}$, with $1.8 \mathrm{X}_{0}$ additional dead material in front of the cryostat. b) Energy distribution with no presampler (solid histogram) and with the optimal presampler weight (open histogram).

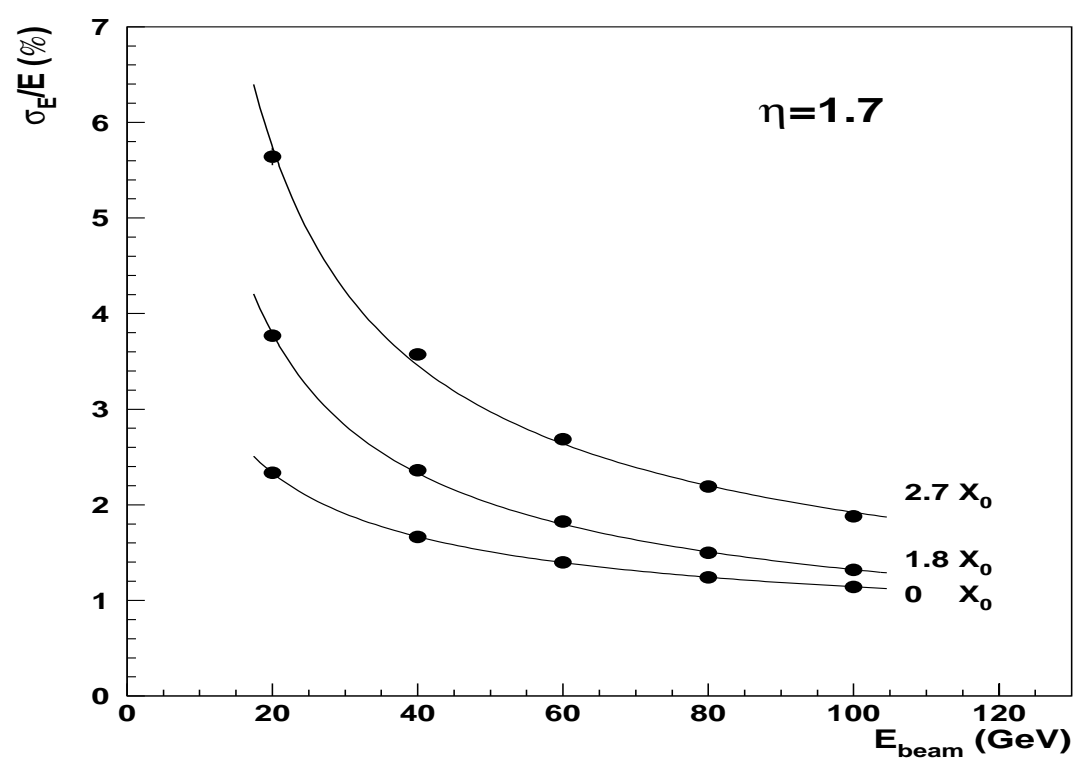

Figure 26: Energy resolution for 3 values of additional dead material as a function of beam energy at $\eta$ $=1.7$ for beam test data. 

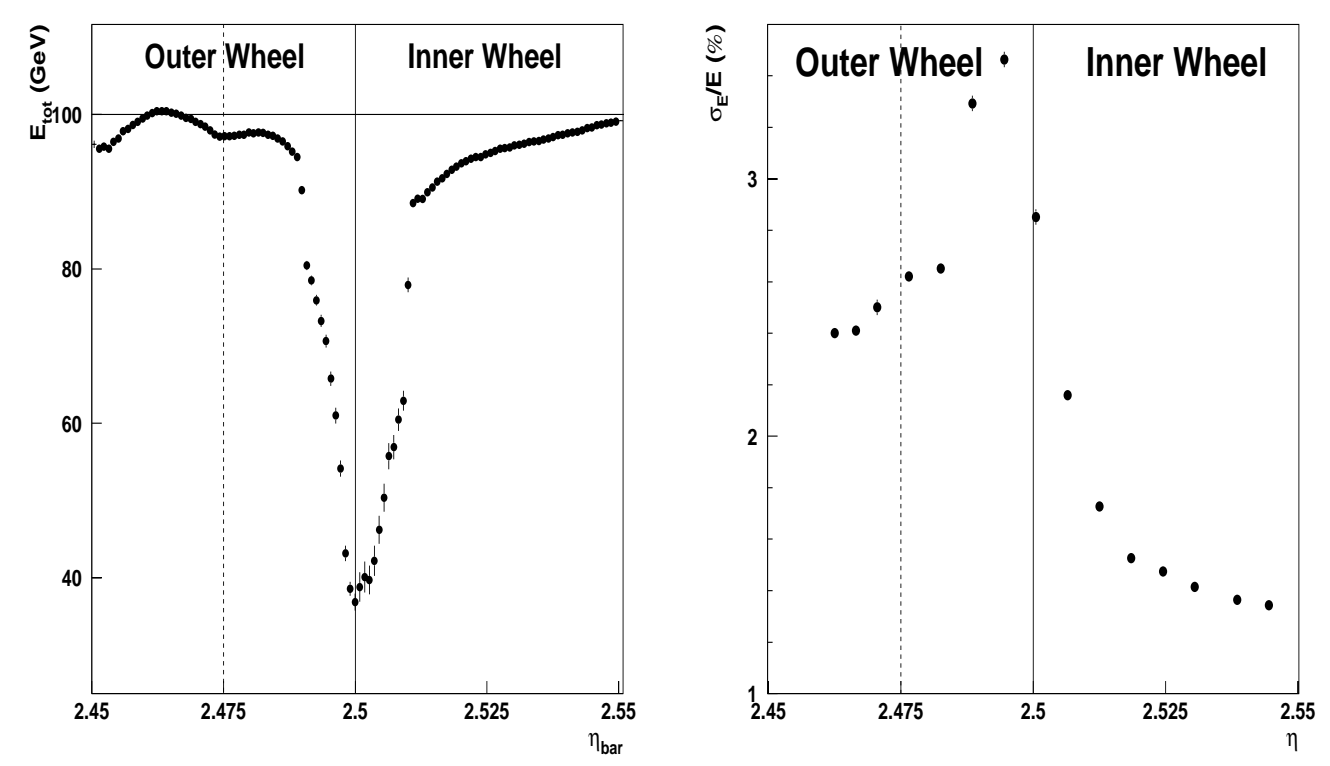

Figure 27: Total energy as a function of $\eta$ barycenter (left) and energy resolution as a function of $\eta$ (right) in the crack region for a $100 \mathrm{GeV}$ electron beam. The vertical dashed lines correspond to the separation between middle cells.

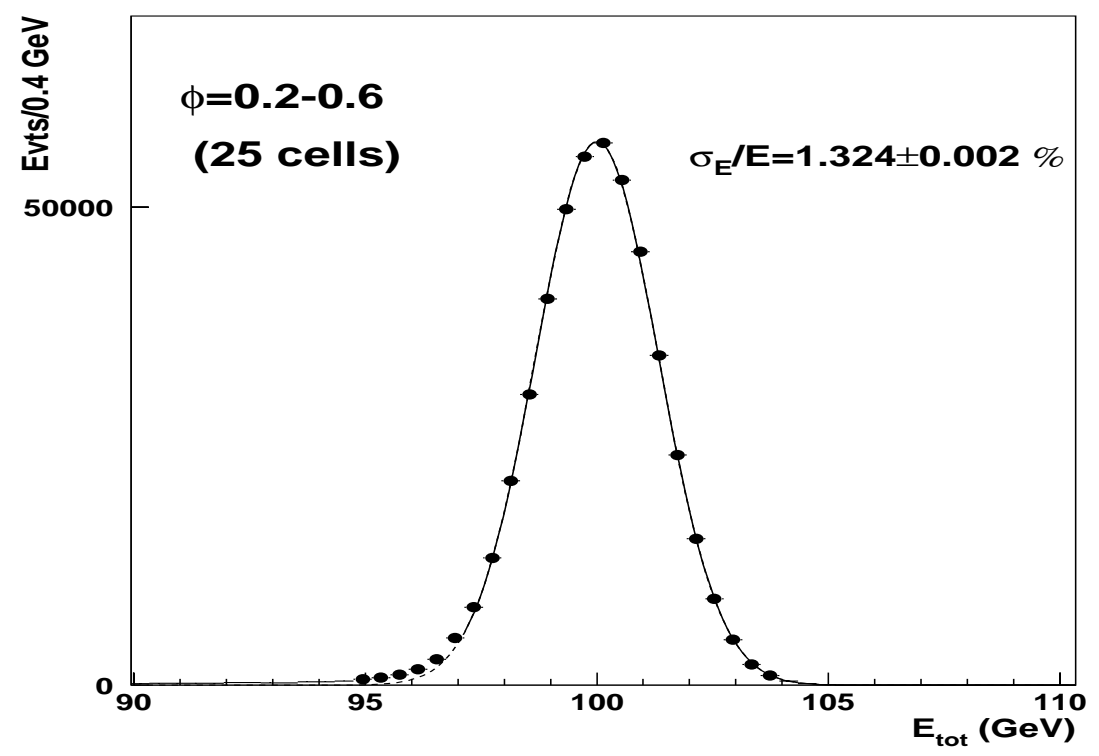

Figure 28: Overall energy distribution reconstructed in the inner wheel for a 100 GeV energy beam. 

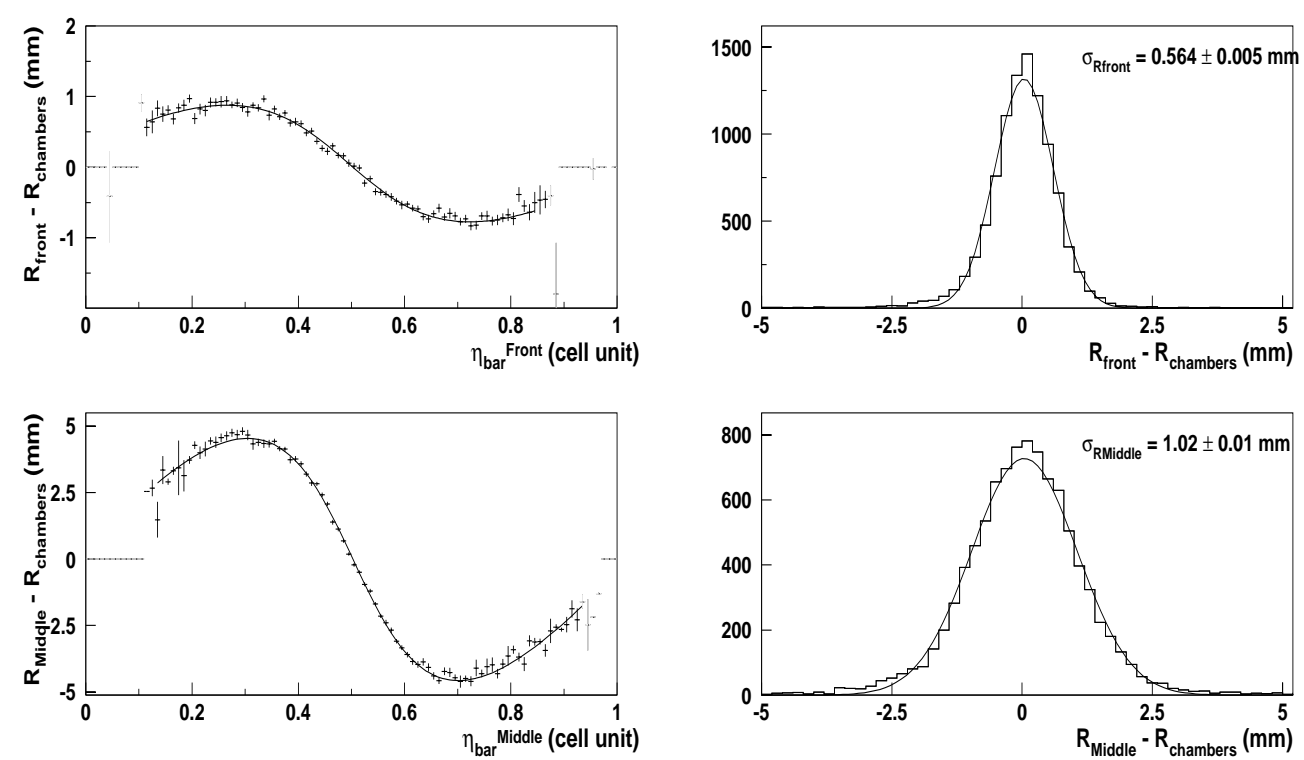

Figure 29: Finite cell size correction (left) and residual fits (right) for $\eta$ barycenters in the front (top) and middle (bottom) section at $\eta=1.9$ and with an electron beam of $40 \mathrm{GeV}$.
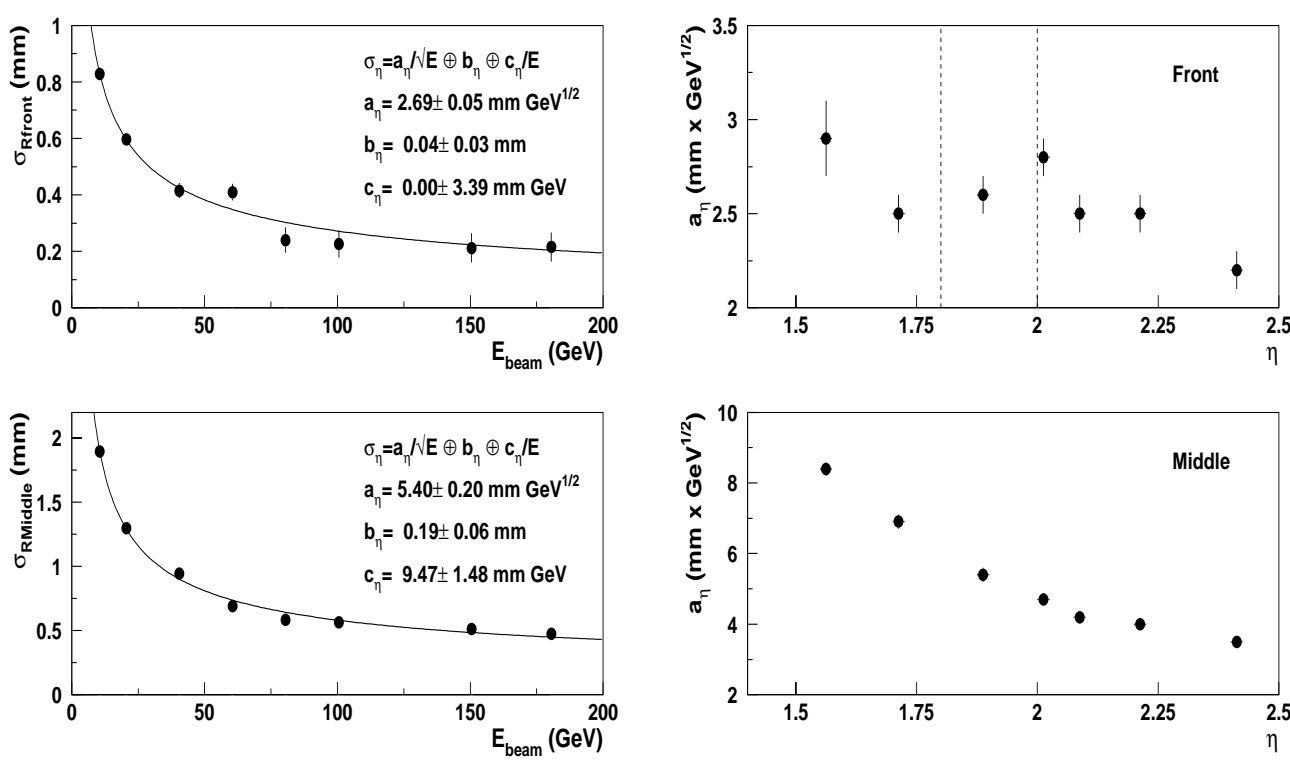

Figure 30: $\eta$ resolution dependence on electron energy at $\eta=1.9$ (left) and stochastic term over all $\eta$ (right), in the front (top) and middle (bottom) section. The vertical dashed lines on the top right plot indicate the change of granularity. 

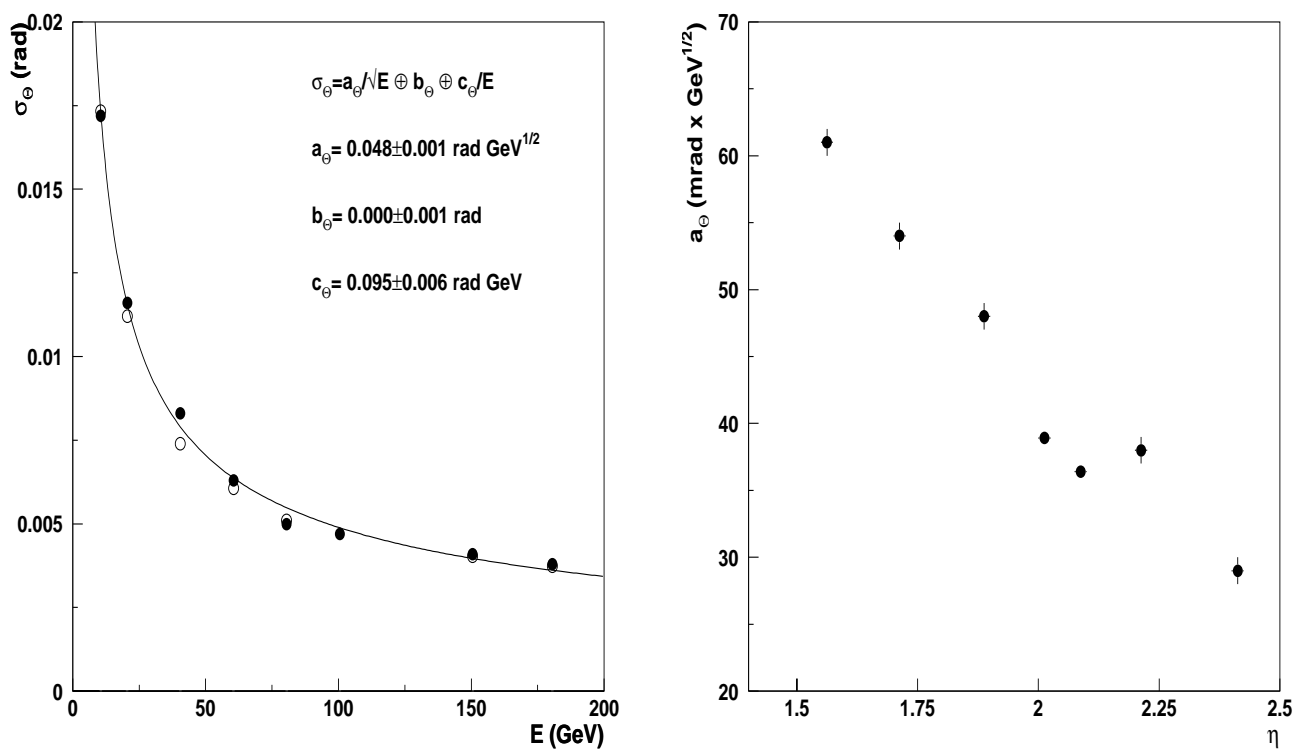

Figure 31: Polar angle resolution at $\eta=1.9$ (left) obtained with beam test data (solid circle) and simulation (open circle). The resolution sampling term, as a function of $\eta$, obtained for beam test data is shown on the right plot. 